Guido Möllering*

\title{
Kartelle, Konsortien, Kooperationen und die Entstehung neuer Märkte ${ }^{* *}$
}

\section{MPIfG Journal Article}

Guido Möllering: Kartelle, Konsortien, Kooperationen und die Entstehung neuer Märkte. In: Schmalenbachs Zeitschrift für betriebswirtschaftliche Forschung 62(7), 770-796 (2010). Verlagsgruppe Handelsblatt/Springer

The original publication is available at the publisher's web site: https://doi.org/10.1007/BF03373680

The MPIfG Journal Articles series features articles by MPIfG researchers and visiting scholars published in peer-reviewed journals. Max Planck Institute for the Study of Societies (MPIfG) Cologne | www.mpifg.de

\section{Zusammenfassung}

Der Beitrag relativiert gängige Vorstellungen von Kooperation als Wettbewerbsverhinderung oder als Reaktion auf Marktversagen und argumentiert, dass Kartelle, Konsortien und andere Formen der Kooperation zwischen Marktakteuren die Voraussetzungen für einen Marktwettbewerb schaffen, der nicht auf den Transaktionswettbewerb zwischen individuellen Marktakteuren beschränkt ist, sondern auch den Wettbewerb zwischen Akteursverbünden (Netzwerkwettbewerb) und den Wettbewerb um Partner (Bindungswettbewerb) einschließt. Gerade in der von hoher Ungewissheit geprägten Marktentstehungsphase kann Strategisches Management durch die Gestaltung von Bindungshorizonten, -intensitäten und -dynamiken die Position eines Unternehmens auf einem neuen Markt wie auch die Entwicklung der konstitutiven Elemente des neuen Marktes insgesamt beeinflussen.

JEL-Classification: L10, L40, Z13.

Keywords: Competition; Cooperation; Markets; Organization; Relationships; Strategy. Bindung; Kooperation; Märkte; Organisation; Strategie; Wettbewerb.

\section{Einleitung: Markterschaffen statt Marktversagen?}

Dieser Beitrag untersucht, welche Rolle verschiedene Formen unternehmensübergreifender Koordination bei der Entstehung neuer Märkte spielen. In der Betriebswirtschaftslehre werden neue Märkte zumeist als neue Absatzchancen verstanden, die sich ein Unternehmen etwa durch Marketingaktivitäten, Produktinnovationen oder Internationalisierung erschließen kann. Insbesondere die institutionenökonomische Perspektive auf neue Märkte betont zwar, dass Märkte von Unternehmen geschaffen werden, versteht darunter jedoch vorwiegend eine Ausweitung des Angebots und die Befriedigung

* Dr. Guido Möllering, Habilitand (extern) an der Freien Universität Berlin, Fachbereich Wirtschaftswissenschaft und Wissenschaftlicher Mitarbeiter am Max-Planck-Institut für Gesellschaftsforschung, Paulstr. 3, 50676 Köln, E-Mail: gm@mpifg.de, Tel.: +49221/2767172, Fax: +49221/2767555.

** Der Autor dankt Dr. Sascha Albers, Prof. Dr. Jens Beckert, Prof. Dr. Jörg Sydow, Dr. Miriam Wilhelm und dem anonymen Gutachter für wertvolle Hinweise und Anregungen. 
latenter Bedürfnisse ${ }^{1}$. Das heißt, es geht lediglich um die Steigerung des eigenen Umsatzes oder Marktanteils. Der jeweilige Markt als unternehmensübergreifendes System wird aus der Sicht des Unternehmens als bereits gegeben vorausgesetz $\mathrm{t}^{2}$. Unternehmen können jedoch nicht nur ihre eigene Position auf bestehenden Märkten optimieren, sie können auch aktiv zu der ursprünglichen Entstehung neuer Märkte beitragen.

Diese strategische Chance steht im Zentrum dieses Beitrags. Es wird betont, dass Unternehmen im Zuge von Marktentstehungsprozessen ihre spätere Marktposition positiv beeinflussen (zum Beispiel im Sinne der „First-Mover Advantages“"3), dass sie sich jedoch auch dem kollektiven Ziel verschreiben, einen neuen Markt überhaupt erst zu erschaffen. Bereits vor 50 Jahren argumentierte Almarin Phillips kurz und grundlegend unter dem Stichwort der „interfirm organization“ für eine stärkere Berücksichtigung der gemeinsamen Interessen der Marktteilnehmer als Gruppe und deren marktstabilisierende Wirkung insbesondere auf Oligopolmärkten ${ }^{4}$. Dabei hatte er jedoch noch nicht die Phase der Marktentstehung im Blick. Die wenigen neueren Forschungen zur aktiven Gestaltung neuer Märkte durch Unternehmen beziehen die kollektive Dimension zwar ein, fokussieren dann jedoch zum Beispiel auf Legitimitäts- und Abgrenzungsfragen, ohne die Möglichkeiten kooperativer Marktgestaltung systematisch zu ergründen5. Auch die einschlägige Literatur zu „Business Webs“6 betont zwar die unternehmensübergreifende Netzwerkebene, verknüpft sie aber meist noch nicht konsequent mit der Marktebene.

In der Phase der Marktentstehung stehen die prospektiven Marktakteure in einem besonderen Verhältnis zueinander. Zwar antizipieren sie bereits die zukünftige Konkurrenz, haben in dieser sogenannten vorwettbewerblichen Phase aber auch ein gemeinsames Interesse an der Schaffung der Voraussetzungen für die Entstehung und Erhaltung des neuen Marktes ${ }^{7}$. Ganz abgesehen davon, ob diese Voraussetzungen eher in einem historischen Prozess spontan emergieren oder vor allem durch regulative Eingriffe organisiert werden ${ }^{8}$, herrscht eine Ungewissheit, die Anbieter und Nachfrager davon abhalten könnte, in neue Märkte zu investieren. Ungewissheit besteht in vielerlei Hinsichten: Zugang zu Ressourcen, Wandel der Umweltbedingungen, Verfügbarkeit von Transaktionspartnern, Gültigkeit von Transaktionsbedingungen, Realisierbarkeit neuer Technolo-

1 Vgl. zum Beispiel Anderson/Gatignon (2005); Jacobides (2005).

2 Hierzu kritisch Aldrich/Fiol (1994), S. 664, die zu einer Beschäftigung mit den Ursprüngen neuer Märkte und Branchen aufrufen („calling attention to the possible origins of a new industry“).

3 Lieberman/Montgomery (1988) definieren First-Mover Advantage als „the ability of pioneering firms to earn positive economic profits“ (S. 41). Der Erfolg solcher Pionierstrategien ist freilich ungewiss.

4 Vgl. Phillips (1960).

5 Vgl. jüngst Santos/Eisenhardt (2009) oder Humphreys (2010) sowie schon Aldrich/Fiol (1994).

6 Vgl. zum Beispiel Franz (2003); Steiner (2004); Schmid (2010); international: Gawer (2009).

7 Vgl. zum Beispiel für neue Halbleitertechnologiemärkte Appleyard et al. (2008). Die Studie von Ingram/Inman (1996) über den Einfluss der Rivalität zwischen Hotels auf der kanadischen und US-amerikanischen Seite der Niagarafälle auf die kollektive Wiederherstellung von Marktvoraussetzungen zeigt ebenso, wie Unternehmen trotz prinzipieller Konkurrenz gemeinsam Märkte möglich machen können.

8 Vgl. hierzu Hayeks (1973), S. 35ff., Unterscheidung zwischen cosmos und taxis. Im vorliegenden Beitrag wird die prinzipielle Gestaltbarkeit von Märkten angenommen, die jedoch empirisch verschiedenen unkontrollierbaren Einflüssen, Zufällen und Dynamiken gegenübersteht. 
gien, Möglichkeit zeitlicher Planung, Zuverlässigkeit des Rechts, Legitimität von Innovationen, Internationalisierungstendenzen und dergleichen ${ }^{9}$.

So verallgemeinert Neil Fligstein: „The real issue for making markets is to create political and social conditions that produce enough stability so as to allow investment " 10 . Die Reduzierung von Ungewissheit ist auch der Ausgangspunkt ökonomischer Institutionentheorien, wie etwa bei Douglass North ${ }^{11}$. Aus einer Managementperspektive vermerken Santos/Eisenhard: „Nascent markets constitute unstructured settings with extreme ambiguity" 12 und sie sehen in dieser Mehrdeutigkeit wohlgemerkt strategische Chancen, die Definition und damit auch die Konstitution neuer Märkte zu beeinflussen. Konzeptionell besonders fruchtbar ist die Anknüpfung an Forschungen zu „negotiated environments“ insbesondere in der Tradition der Resource-Dependence-Theorie ${ }^{13}$. Dort wird nämlich die Etablierung interorganisationaler Beziehungen als eine Möglichkeit der Reduktion von Ungewissheit gesehen. Christine Oliver etwa behauptet: „Uncertainty prompts organizations to establish and manage relationships in order to achieve stability, predictability, and dependability in their relations with others" ${ }^{14}$. Dieses lässt sich auch auf die Ungewissheit in Marktentstehungsphasen übertragen, in denen durch unternehmensübergreifende Kooperation Ungewissheit reduziert und absorbiert werden kann, ohne jedoch jemals vollständig eliminiert zu werden. Ein aktives Engagement im Sinne der „negotiated environments“ stellt ein eigenes unternehmerisches Handlungsfeld des Strategischen Managements mit einer eher langfristigen und explorativen Ausrichtung im Spannungsfeld von Kooperation und Wettbewerb dar ${ }^{15}$.

Damit wird hier eine Thematik aufgegriffen und konzeptionell erweitert, der sich bereits die 61. Jahrestagung des Verbandes der Hochschullehrer für Betriebswirtschaft 1999 in Bamberg widmete ${ }^{16}$. Damals wurde nur angedeutet, dass Kooperation nicht nur als Alternative zum Wettbewerb zu sehen ist, sondern dass auch eine Wechselwirkung zwischen Unternehmenskooperationen und deren Wettbewerbsumfeld besteht. Der vorliegende Beitrag setzt den Akzent genau darauf, wie Kooperation Wettbewerb - und damit Märkte - ermöglicht. Der hier umrissene Ansatz lässt die transaktionskostentheoretische (Hilfs-)Annahme der Existenz von Märkten als Urzustand, von dem

9 Hierzu vgl. u.a. Cook (1977); Aldrich/Fiol (1994); Dickson/Weaver (1997); Appleyard et al. (2008). Wohlgemerkt handelt es sich hierbei nicht um (berechenbares) Risiko, sondern um (unberechenbare) „uncertainty“ nach Knight (1971[1921]).

10 Fligstein (2001), S. 23.

11 Vgl. North (1990).

12 Santos/Eisenhard (2009), S. 644.

13 Vgl. v.a. Pfeffer/Salancik (1978) und dort zu „negotiated environment“ S. 143ff., wo der Begriff in Anlehnung an Cyert/March (1963) eingeführt wird.

14 Oliver (1990), S. 245f. Vgl. auch Cook (1977); Podolny (1994); Dickson/Weaver (1997). Speziell für Gemeinschaftsunternehmen konstatieren Pfeffer/Salancik (1978), S. 154, dass ,joint ventures are undertaken to reduce uncertainty and promote stability in the environment".

15 Vgl. auch das Konzept der strategischen Institutionalisierung von Ortmann/Zimmer (1998), das die Autoren allerdings vor allem für die Analyse des Verhältnisses zwischen staatlicher Regulation und unternehmensseitiger Einflussnahme in Anschlag bringen („Rekursive Regulation“).

16 Vgl. Engelhard/Sinz (1999). 
nur im Falle des Marktversagens abgewichen wird, in einem neuen Lichte erscheinen ${ }^{17}$. Damit neue Märkte entstehen können, bedarf es hierarchisch koordinierter Unternehmen, die Ressourcen bündeln und entwickeln und die auch miteinander kooperieren, um die Voraussetzungen für Marktentstehung zu schaffen.

Gleichwohl werden die Gefahren wettbewerbsbeschränkender Strukturen und Aktivitäten auf neuen Märkten nicht geleugnet ${ }^{18}$. Es gilt zu verstehen, wann und wie Kooperation neue Konkurrenz ermöglicht und sie auf Dauer eben nicht (zu sehr) unterbindet ${ }^{19}$. Die folgenden Überlegungen beziehen auch die volkswirtschaftliche Marktphasentheorie nach Ernst Heuß ein, welche die Besonderheiten der sogenannten Expansionsphase in der Entstehung eines Produktmarktes und die dafür typische hohe Ungewissheit und ständige Veränderung anerkennt. Allerdings spielt nicht erst in den reiferen Marktphasen Kooperation „zwangsläufig“20 eine strategische Rolle, sondern bereits weit davor, weil Kooperationen dazu beitragen, Ungewissheit so weit zu reduzieren, dass Marktwettbewerb möglich wird und ein Markt entstehen kann ${ }^{21}$.

\section{Wirkungen unternehmensübergreifender Koordination auf die Entstehung neuer Märkte}

\subsection{Marktkonstitution: Elemente und Prozesse}

Märkte sind gekennzeichnet durch vorwiegend über den Preis koordinierte Transaktionen zwischen Anbietern und Nachfragern, die um günstige Transaktionsmöglichkeiten und -bedingungen konkurrieren. Diese Marktdefinition erfasst den Kern des abstrakten ökonomischen Marktbegriffs, impliziert zugleich die empirische Realität verschiedenartiger Märkte und lehnt sich dabei an Konzepte von Max Weber an ${ }^{22}$. Damit ein Markt als abgrenzbares Transaktionssystem ${ }^{23}$ konstituiert und empirisch erkennbar wird, müssen sich verschiedene Elemente spezifisch herausbilden, und zwar die Produkte, Transaktionsformen, Informationen, Akteure (Anbieter, Nachfrager, Intermediäre und Regulatoren), Netzwerke und Institutionen des jeweiligen Marktes (vgl. Abbildung 1) ${ }^{24}$.

Von einem neuen Markt kann die Rede sein, wenn sich eines oder mehrere der Elemente signifikant wandeln und somit Transaktionen zustande kommen, die nicht mehr

17 Vgl. Williamson (1975), S. 20: „I assume, for expositional convenience, that in the beginning there were markets'“. Vgl. hierzu auch kurz und kritisch Ortmann (2005). Zum Stichwort „Marktversagen“ vgl. auch zum Beispiel Schauenberg (2004).

18 Auch Pfeffer/Salancik (1978), S. 183f., übersehen keinesfalls, dass Kartelle Dritte benachteiligen können: "Only a few members of a market may be participating and it is frequently the least powerful and the least organized whose interests are not served in the resultant organizational structure."

19 Vgl. Teece (1992).

20 Sjurts (2000), S. 35.

21 Zum Zusammenhang von Marktphase und funktionsfähigem Wettbewerb vgl. Zohlnhöfer (1991).

22 Vgl. Weber (1980[1922]) S. 43ff., 382ff., insbes. auch dessen Rede von „Marktkampf“, S. 58.

23 Die Rede von Markt als System (genauer: Transaktionssystem) ist hier angelehnt an Biggart/Delbridge (2004) und deren Typologie verschiedener "Systems of Exchange“.

24 Vgl. für eine genauere Darstellung marktkonstituierender Elemente Möllering (2009a). 
einem bereits bestehenden Markt zugeordnet werden können. Das bedeutet, dass nicht nur Produktinnovationen, sondern auch neuartige Transaktionsformen (zum Beispiel Online-Handel), Standards (zum Beispiel Blue Ray), Zertifizierungen (zum Beispiel Fair-Trade-Siegel), Akteursgruppen (zum Beispiel Solarinitiativen), Beziehungsmuster (zum Beispiel Kundenkarten) oder Regeln (zum Beispiel mögliche Aufhebung des Fremdbesitzverbotes von Apotheken) jeweils einen „neuen“ Markt begründen können. Eher hypothetisch wäre der Fall eines gänzlich neuen Marktes, in dem sich alle Elemente zugleich aus dem Nichts herausbilden.

\section{Abbildung 1: Konstitutive Marktelemente (Übersicht)}

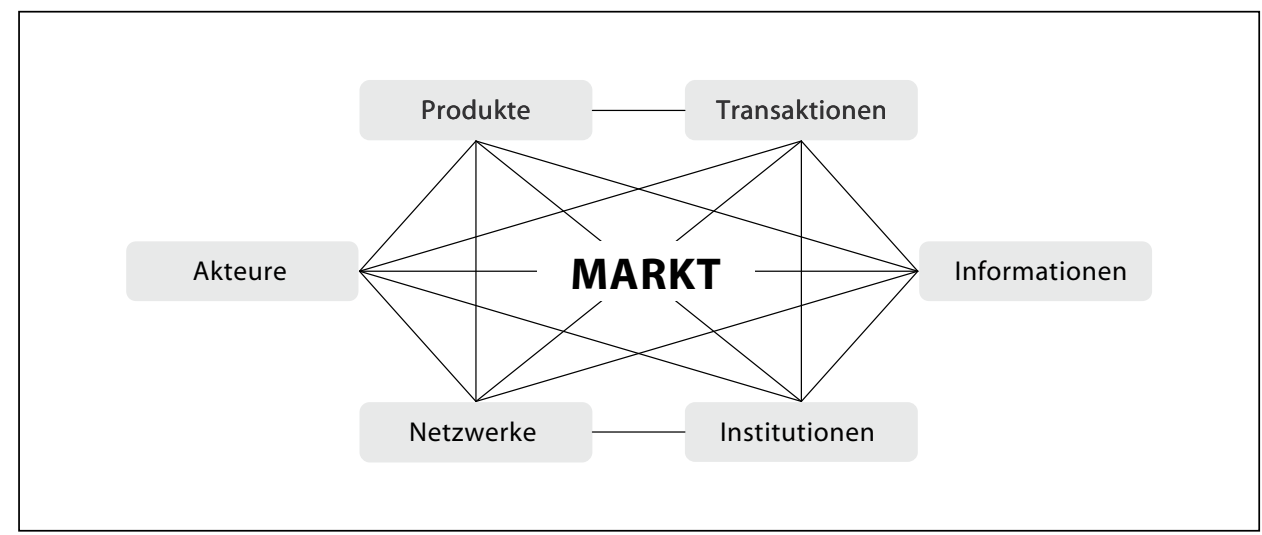

Erläuterung: Ein Markt als Transaktionssystem wird durch die hier dargestellten, sich wechselseitig beeinflussenden Elemente konstituiert. Jeder empirische Markt hat Eigenheiten in seinen Akteuren, Produkten, Transaktionen, Informationen, Netzwerken und Institutionen (Quelle: Möllering (2009a), S. 11).

Die Entstehung eines Marktes vollzieht sich mithin in Konstitutionsprozessen, in denen die Elemente des neuen Marktes geformt werden. Produkte entstehen durch Innovation, Transaktionsformen durch Standardisierung, Informationsgrundlagen durch Kommunikation, Marktakteure durch Wettbewerbsverhalten, Netzwerke durch Bindungen und Institutionen durch die Etablierung von Regeln. Letzteren kommt eine besondere Bedeutung zu. So listet Elinor Ostrom zwar allgemeine Regeln für einen offenen Wettbewerbsmarkt auf, verweist aber zugleich auf empirisch variable „working rules“ und „rule configurations“ in Märkten ${ }^{25}$. Dass jeder Markt spezielle Regeln hat, kommt auch in Neil Fligsteins Begriff der „conceptions of control“ zum Ausdruck, der die institutionellen Konfigurationen realer Märkte in der Wahrnehmung von Managern bezeichnet ${ }^{26}$. Die Eigenheiten empirischer Märkte werden besonders deutlich von Michel Callon betont, der 
meint: „It is wrong to talk of laws ... of the market. There exist only temporary, changing laws associated with specific markets" 27 . Innerhalb dieses abstrakten Bezugsrahmens stellt sich die praktische Frage, inwieweit Unternehmen individuell (zum Beispiel durch Produktentwicklung, Vertriebsinnovationen oder Lobbying) und auch kollektiv Einfluss auf Marktelemente und deren Herausbildungsprozesse nehmen können ${ }^{28}$. Diese Möglichkeiten sollen im Folgenden genauer differenziert werden.

\subsection{Kartelle: Inkubatoren und Inhibitoren für neue Märkte}

Kartelle sind der Inbegriff der Wettbewerbsbeschränkung durch Kollusion ${ }^{29}$. Preisabsprachen sowie auch die gebietsmäßige oder anderweitige Aufteilung von Markträumen unter Anbietern (bzw. auch Nachfragern) reduzieren den Konkurrenzdruck. Kartelle haben definitionsgemäß das Ziel und empirisch den Effekt, den Wettbewerb zu unterbinden, der doch charakteristisch und notwendig für Märkte ist. Allerdings sieht das Wettbewerbsrecht nicht nur in Deutschland und der Europäischen Union die Möglichkeit der Tolerierung oder gar Genehmigung von Kartellen vor, soweit diese dem Allgemeinwohl dienen oder nicht spürbar wettbewerbsbeschränkend wirken ${ }^{30}$. So folgt ja auf das Kartellverbot in $\$ 1$ GWB sogleich der Freistellungsparagraph $\$ 2$ GWB. Allgemein sind Kartelle das kleinere Übel, wenn ohne sie zum Beispiel die Versorgung mit wichtigen Gütern zusammenbräche, wenn etwa die Angebotsseite ohne Kartell wirtschaftlich unattraktiv würde und mit den Anbietern der gesamte Markt verschwände.

Speziell auf die Entstehung neuer Märkte bezogen lautet die kartellfreundliche Argumentation wie folgt: Bei dem anfangs noch geringen und ungewissen Marktvolumen eines neuen Marktes lässt man eine Einschränkung des Wettbewerbsdrucks zu, damit sich eine kritische Masse von Anbietern herausbilden kann, für die der Markt attraktiv genug ist, um entsprechende Investitionen zu tätigen ${ }^{31}$. Kartelle wirken dann wie ein Inkubator für Marktakteure, die wiederum durch Innovationen und andere Aktivitäten der Marktgestaltung (zum Beispiel Vereinbarung von Standards) den neuen Markt schaffen ${ }^{32}$.

27 Callon (1998), S. 47.

28 Vgl. u.a. Aldrich/Fiol (1994). Osborn/Hagedoorn (1997), S. 272, bemerken hierzu: „[Alliances and networks] may solve economic, technical, and strategic problems for their sponsors as they also develop, produce, and market goods, services, and knowledge."

29 Vgl. vor dem Hintergrund der Unternehmenskooperation für die Betriebswirtschaftslehre Schubert/Küting (1981), S. 142ff., und für die Rechtswissenschaft Lange (1998), S. 455ff., und Kirchner (1999).

30 Lange (2001), S. 289, verweist auf eine gängige Praxis auf EU-Ebene, nach der keine Spürbarkeit angenommen wird, wenn bei horizontalen Vereinbarungen weniger als 5\% der Marktanteile und bei vertikalen Vereinbarungen weniger als 10\% der Marktanteile betroffen sind, vgl. auch Lange (1998), S. 466ff. Zur wettbewerbspolitischen Auslegung der Vor- und Nachteile von Kartellen vgl. von Weizsäcker (1999).

31 Dies entspricht der unternehmerischen Strategie junger Unternehmen, wie etwa bei Santos/Eisenhardt (2009), S. 653ff. beschrieben, die eigene Position in einem entstehenden Markt durch Bindungen an bereits etablierte Marktakteure zu stabilisieren - und zugleich die mächtigeren Akteure zu zähmen.

32 Für die Beurteilung von Kartellen ist ein zentrales Kriterium, welche Marktanteile durch ein Kartell kontrolliert werden. Bei neu entstehenden Märkten ist dieses Kriterium jedoch problematisch, da das Marktvolumen noch klein ist und monopolartige Stellungen der frühen Marktteilnehmer instabil sind. 
Aus der gleichen Logik leitet sich jedoch auch ab, dass Kartelle nicht mehr funktional (und auch nicht mehr wettbewerbspolitisch vertretbar) sind, wenn der Markt eine hinreichende Größe und Stabilität erreicht hat. Kartelle sind dann nicht mehr Inkubatoren, sondern Inhibitoren für die weitere Marktentwicklung und insbesondere für den Eintritt neuer Marktakteure. In einer marktwirtschaftlichen Ordnung müssen dann die dafür zuständigen Behörden eingreifen und bestimmte Kartelle verbieten, die zuvor zugelassen oder toleriert wurden. Außerdem können sich die Kartelle selbst auflösen, wenn ihre Mitglieder sie nicht mehr für nötig oder vorteilhaft halten. Die mit einem Kartell verbundenen Wettbewerbsbeschränkungen bedeuten ja nicht nur Nachteile für Kunden und Eintrittsbarrieren für Neueinsteiger, sondern können auch die etablierten und zugleich kartellierten Marktakteure in ihren Entwicklungsmöglichkeiten behindern. Gerade die Option der Auflösung und des Bruches von Kartellen verdeutlicht, dass in ihnen stets noch der Wettbewerb latent vorhanden ist ${ }^{33}$. Gleichwohl ist davon auszugehen, dass frühere Kartelle die Strukturen eines neuen Marktes noch lange weiterprägen ${ }^{34}$.

\subsection{Konsortien: Risikostreuung, Ressourcenbündelung und Routinisierung}

Konsortien sind Gelegenheitsgesellschaften, in denen sich rechtlich und wirtschaftlich selbstständige Unternehmen zusammenschließen, um eine zeitlich befristete und inhaltlich abgegrenzte Aufgabe gemeinschaftlich zu lösen ${ }^{35}$. Folglich stimmen die Konsorten ihre Verhaltenweisen ähnlich wie in Kartellen ab. Allerdings verbindet man mit dem Begriff des Konsortiums deutlich weniger das Problem der Wettbewerbsbeschränkung. Das liegt zum einen daran, dass Konsortien nicht unbedingt zwischen Wettbewerbern gebildet werden, sondern zwischen Unternehmen, deren Angebote auf verschiedenen Märkten sich ergänzen können, wenn zum Beispiel in der Bauindustrie Unternehmen aus verschiedenen Gewerken als Arbeitsgemeinschaft ein Objekt errichten ${ }^{36}$. Aber auch wenn Konsortien aus horizontalen Wettbewerbern bestehen (typisch etwa bei Emissionskonsortien im Bankwesen) oder vertikal eine exklusive Zusammenarbeit entlang einer Wertkette beinhalten (zum Beispiel Logistik-Konsortien zur Einführung von RFID-Technologien im Handel), so steht dabei nicht die Wettbewerbsbeschränkung im Vordergrund. Vielmehr versetzen sich die Konsorten durch ihre Zusammenarbeit in die Lage, gemeinsam als Marktakteur auf einem Markt aktiv zu werden, auf dem sie alleine nicht als Anbieter oder Nachfrager auftreten könnten (zum Beispiel KMU-Konsortien als Konkurrenten zu Baukonzernen) ${ }^{37}$. Somit sind Konsortien für diesen Markt eher wettbewerbsförderlich. Zudem wirken sie wegen ihrer grundsätzlichen Befristung und inhaltlichen Abgrenzung eher punktuell auf den Markt.

33 Vgl. hierzu Das/Teng (2000), allerdings mit Bezug auf Strategische Allianzen.

34 Stack/Gartland (2005) etwa zeigen, dass große amerikanische Brauereien dauerhaft von den sie begünstigenden und durch sie selbst mitgestalteten Wettbewerbsregeln nach Aufhebung der Prohibition profitierten - und zwar auch noch, nachdem man diese Regeln später zu ihren Ungunsten revidierte.

35 Vgl. die Definition bei Wöhe/Döring (2008), S. 261, sowie grundlegend Schubert/Küting (1981), S. 104ff., sowie zur juristischen Bedeutung von Konsortien Steding (2004).

36 Vgl. Schubert/Küting (1981), S. 108f.

37 Vgl. zum Beispiel Kirchner (1999), S. 448. 
In der Entstehung neuer Märkte können Konsortien, ähnlich wie Kartelle, die Herausbildung neuer Marktakteure und deren Innovations- und Institutionalisierungsbestrebungen fördern. Während Kartelle jedoch eher dem einzelnen Unternehmen einen gewissen Schutzraum sichern, dienen Konsortien dem gemeinsamen Handeln mehrerer Unternehmen, die sodann im Konsortium als Anbieter oder Nachfrager kollektiv einen Markt erschließen. Vor dem Hintergrund der hohen Investitionsunsicherheiten in neuen Märkten erfüllen Konsortien vor allem die Funktionen der Risikostreuung und der Ressourcenbündelung. Die Konsorten addieren nicht nur ihre finanziellen Ressourcen, sondern kombinieren auch ihr Wissen aus unterschiedlichen Bereichen, die für den neuen Markt relevant sind. Während die Funktionen von Konsortien im Allgemeinen recht klar sind, ist die Organisation und Führung von Konsortien nur spärlich erforscht ${ }^{38}$. Gerade von diesem „Innenleben“ der Konsortien hängt jedoch ab, ob sie nachhaltige Wirkungen auf neue Märkte ausüben.

Empirisch interessant ist vor allem die Frage, inwieweit Konsortien zu einer Stabilisierung des Marktes führen. Zum einen ist vorstellbar, dass einzelne Konsorten, und zwar insbesondere die Konsortialführer, sich aus Konsortien heraus als Wettbewerber auf dem neuen Markt etablieren. In diesem Fall wirkt das Konsortium, ähnlich wie bei Kartellen möglich, als Inkubator. Dabei kann es auch zu (teilweisen) Akquisitionen, Fusionen oder der Gründung von Gemeinschaftsunternehmen kommen. Zum anderen ist zwar jedes Konsortium zeitlich und inhaltlich begrenzt, doch Unternehmen können immer wieder neue Konsortien bilden, dabei bevorzugt mit früheren Konsorten arbeiten und einen Großteil der Geschäfte auf dem neuen Markt über Konsortien tätigen ${ }^{39}$. In diesem Fall rücken Konsortien konzeptionell in die Nähe von Projektnetzwerken, die ja dadurch gekennzeichnet sind, dass zwischen temporärem Projektgeschäft und projektübergreifenden Beziehungen ein rekursiver Zusammenhang besteht ${ }^{40}$.

Für neue Märkte bedeutet dies tendenziell - im Vergleich zu einer Konsolidierung einiger weniger Großanbieter und -nachfrager - eine größere Dynamik. Allerdings können auch in seriellen Konsortien (mit wiederkehrenden Konsorten) und in Projektnetzwerken Pfadabhängigkeiten entstehen, die zum einen wie Markteintrittsbarrieren für neue Marktakteure wirken und zum anderen auch die Entwicklungsmöglichkeiten der Insider beschränken ${ }^{41}$. Ähnlich zwiespältig wären Konsortien zu sehen, die ihren zeitlichen Horizont und ihre inhaltliche Aufgabe immer weiter ausweiten, gar bis hin zu einer völlig offenen Ausrichtung, die dann zwar die Beziehungen wichtiger Marktakteure auf Dauer stabilisiert, dabei aber kein Konsortium mehr konstituiert, sondern eine feste Allianz, die den Markt in seiner Entwicklung längerfristig prägen könnte.

Eine spezielle Ausprägungsform von Konsortien mit einer solchen dauerhaft strukturierenden Wirkung stellen Business Webs dar. In ihnen gehen Unternehmen kooperative Beziehungen ein, um eine (meist) technologische Plattform zu entwickeln, sie

38 Vgl. Möllering (2009b), S. $592 \mathrm{f}$.

39 Vgl. Li/Rowleys (2002) Untersuchung über Emissionskonsortien zwischen U.S.-Investment Banken.

40 So etwa Corsten (2003), S. 55, und Wohlgemuth (2002), S. 24. Zu Projektnetzwerken im Allgemeinen vgl. Sydow/Windeler (1999), insbes. S. 217 (Definition).

41 Vgl. zum Beispiel Powell (1998); Li/Rowley (2002); Kim/Oh/Swaminathan (2006). 
möglichst als Standard zu etablieren und mit einem vielfältigen Angebot an Komplementärleistungen zu stützen ${ }^{42}$. Aus Business Webs und der Konkurrenz zwischen ihnen können neue Märkte entstehen, deren Wettbewerbsintensität und Dynamik jedoch genau durch jene Dominanz eingeschränkt werden könnte, welche die an Business Webs beteiligten Unternehmen zu erreichen suchen ${ }^{43}$.

\subsection{Kooperationen: Entwickeln, Lernen und Binden}

Auch Kartelle und Konsortien beruhen auf kooperativen Mechanismen, jedoch weisen sie in der Regel eine nur mittlere bis niedrige Bindungsintensität auf und basieren mehr auf der Koordination der eigenständigen Aktivitäten der beteiligten Unternehmen als auf einer engeren Verknüpfung und Verschränkung von Aktivitäten. Kooperationen sollen daher im Folgenden enger als eine partnerschaftliche Koordinationsform verstanden werden, bei der Unternehmen die eigenen Leistungsprozesse wesentlich auf die Leistungsprozesse ihrer Partner einstellen, von diesen teilweise abhängig machen und gemeinsam mit den Partnern entwickeln. Die Zusammenarbeit ist hier mithin recht intensiv, die Investitionen sind spezifisch, die Partner sind weniger austauschbar und die Risiken sind nicht nur gestreut, sondern vor allem auch verquickt. Trotz der eher hohen Bindungsintensität müssen Kooperationen nicht immer langfristig angelegt sein (wie zum Beispiel bei Strategischen Allianzen). Sie können auch eher kurz und intensiv sein (zum Beispiel modellbezogene Systempartnerschaften, gemeinsame F\&E-Programme).

In der Entstehung neuer Märkte können Kooperationen alle konstitutiven Marktelemente beeinflussen (vgl. Abbildung 1 oben). Sie dienen dabei intendiert - aber auch unintendiert - etwa der Produktentwicklung, der Etablierung von Produktstandards und Vertriebswegen, der Kompetenzentwicklung der Marktakteure, der Herausbildung von Statusunterschieden, der Abgrenzung von Lieferantenbasen und Kundenstämmen sowie der Klärung von spezifischen Wettbewerbsregeln (das heißt auch: Kooperationsregeln) für einen neuen Markt ${ }^{44}$. Die Ungewissheit, die einer Marktentstehung entgegenstehen könnte, wird reduziert, indem durch Kooperationen eingegrenzt wird, wer was wie mit wem handelt. Durch diese Strukturierung wird der Markt erst als solcher erkennbar und werden die Akteure in ihm handlungsfähig ${ }^{45}$. Die Kooperation soll und darf den Wettbewerb jedoch nicht verdrängen ${ }^{46}$. Man unterscheidet daher - nicht zuletzt in wettbewerbsrechtlichen und wettbewerbspolitischen Erwägungen - vorwettbewerbliche Kooperationen, die (noch) nicht in direktem Zusammenhang mit Markttransaktionen stehen, von kooperativen Marktbeziehungen, die den Wettbewerb insoweit einschränken, als es enge Kunden- und Lieferantenbeziehungen gibt, die ihn aber auch nicht völlig ausschalten, da der Kunden- oder Lieferantenwechsel prinzipiell möglich bleibt. 
Für die Marktentstehung besonders wichtig sind interorganisationale Lernprozesse, die in Kooperationen stattfinden und den Wettbewerb im Markt beeinflussen ${ }^{47}$. Kooperationspartner lernen etwas übereinander, voneinander und miteinander. In vertikalen Beziehungen in einem neuen Markt können Kunden und Lieferanten das, was sie jeweils nachfragen und anbieten, erst einmal konkretisieren und aufeinander abstimmen. Kunden lernen vom Lieferanten, was sie kaufen könnten, und Lieferanten lernen von ihren Kunden, was sie anbieten sollten. Jede Seite stellt sich auf die andere ein. Beide Seiten lernen jedoch auch, wie sie die Transaktionsbedingungen zu ihren eigenen Gunsten beeinflussen können, und dies prägt sodann den vertikalen Wettbewerb um einen möglichst guten „Deal“.

Außerdem können die Unternehmen ihr Wissen aus einer vertikalen Kooperation mit einem bestimmten Partner einsetzen, wenn sie mit dessen horizontalen Konkurrenten Geschäfte machen, sodass dieses Wissen auch den horizontalen Wettbewerb beeinflusst. Sogenannte „races to learn“ 48 sind ein bekanntes Phänomen, in dem zum Ausdruck kommt, dass kooperiert wird, (nur) solange man von dem Partner etwas lernt, das die eigene Wettbewerbsposition insgesamt verbessert. Auch in horizontalen Kooperationen mit Konkurrenten auf der gleichen Stufe lernen Unternehmen zunächst viel über sich selbst sowie über ihre Partner und andere Wettbewerber. Dieses Wissen nutzen sie außerhalb der Kooperation oder nach deren Beendigung aus, wenn sie um günstige Transaktionen konkurrieren. Auch in diesem Sinne schaffen Kooperationen Voraussetzungen für Wettbewerb, und zwar, indem sie kompetente Marktakteure produzieren.

Jedoch ist es nicht immer zwingend, dass Kooperationen nur vorübergehend und zur opportunistischen Verbesserung allein der eigenen Wettbewerbssituation eingegangen werden. Kooperationspartner können auf Märkten auch dauerhaft miteinander lernen, geringes Interesse an Wechseloptionen haben und stattdessen als Gespann bessere Ergebnisse als die Konkurrenz anstreben. Dies wäre etwa typisch für Business Webs. Allerdings können sehr enge Verflechtungen zwischen Marktakteuren auch zu Pfadabhängigkeiten führen, sodass es den betroffenen Unternehmen unmöglich werden kann, aus einer Beziehung auszubrechen, wenn sie nicht mehr attraktiv ist. Die Gründe hierfür können vertraglicher Natur sein ${ }^{49}$, aber auch in fundamentaleren (zum Beispiel technologischen) Pfadabhängigkeiten in den beteiligten Organisationen ${ }^{50}$ sowie auf der Beziehungs- und Netzwerkebene liegen ${ }^{51}$. Und dies bedeutet wiederum eine eingeschränkte Wettbewerbsintensität in dem Markt, zumal wenn viele Marktakteure derart enge Beziehungen unterhalten ${ }^{52}$. Bei der Entstehung eines Marktes besteht daher die Gefahr, dass Kooperationen, die zunächst ein wettbewerbsförderliches Lernen ermöglichen, ab einem gewissen Punkt der Marktentwicklung abträglich werden. Bei aller nötigen kooperativen Reduzierung von Ungewissheit müssen die Anbieter und Nach-

47 Vgl. Kogut (1988); Osborn/Hagedoorn (1997), S. 269ff.

48 Hamel (1991), S. 85.

49 Vgl. Lange (1998).

50 Vgl. Li/Rowley (2002).

51 Vgl. Semlinger (1993); auch Bresser/Harl (1986).

52 Zur „overembeddedness“ vgl. zum Beispiel Uzzi (1997), S. 58ff.; Hagedoorn/Frankort (2008), S. $510 \mathrm{ff}$. 
frager sich trotz aller (quasi-)monopolistischen Anreize als Wettbewerber verstehen und verhalten - und sei es, wie im nächsten Abschnitt betrachtet wird, als Teil eines Netzwerks im Wettbewerb mit anderen Netzwerken.

\subsection{Netzwerkwettbewerb auf neuen Märkten?}

Dem positiven Beitrag zur Entstehung eines neuen Marktes, den Kartelle, Konsortien und engere Kooperationen leisten können, steht jeweils die Gefahr gegenüber, dass sich die Bindungen zwischen Unternehmen so stark verfestigen, dass sie in dem später dann etablierten Markt den Wettbewerb behindern und damit auch die längerfristig wünschenswerte Dynamik und Offenheit des Marktes einschränken ${ }^{53}$. Ob dies eintritt, hängt davon ab, wie viele kooperative Verbünde in einem Markt bestehen und wie diese zueinander stehen. In einigen Märkten, wie etwa den Automobil- und Luftfahrtmärkten, wird beobachtet, dass der Wettbewerb nicht mehr vorrangig zwischen Einzelunternehmen, sondern zwischen unternehmensübergreifenden Allianzen und Supply Networks ausgetragen wird ${ }^{54}$. Einige neue, stark technologisch getriebene Märkte funktionieren fast ausschließlich über Business Webs, die zwar häufig über ein zentrales Unternehmen (den sog. Shaper) identifizierbar sind, aber erst durch weitere Unternehmen (sog. Adapter) zu einem Netzwerk werden, das mit anderen Business Webs konkurriert ${ }^{55}$.

In solchen Netzwerk-Oligopolen kann der Wettbewerb sehr intensiv sein. Es gibt in ihnen kein den Markt überspannendes Kartell und auch die Etablierung eines dauerhaften Standards ist vor allem ein strategisches Ziel, aber kein zwangsläufiges Ergebnis. Auch die Tatsache, dass Innovationen heutzutage zumeist in Netzwerken entstehen ${ }^{56}$, spricht dafür, dass die unternehmensübergreifende Koordination der Aktivitäten von Marktakteuren der Dynamik des jeweiligen Marktes zuträglich ist. Die miteinander im Wettbewerb stehenden Netzwerke müssen als Netzwerke innovativ bleiben, um zu bestehen („cooperating to compete“) 57 . Neuere Forschungen belegen zudem, dass auch innerhalb prinzipiell kooperativer Netzwerke der Wettbewerb bestehen bleibt, denn die Partner müssen sich auf ihrem Platz im Netzwerk bewähren und konkurrieren ständig auch mit Unternehmen innerhalb und außerhalb des Netzwerks, die ihren Platz einnehmen könnten („competing to cooperate“) ${ }^{58}$.

53 Vgl. zum Beispiel Powell (1998); Li/Rowley (2002); Kim/Oh/Swaminathan (2006).

54 Den Wettbewerb zwischen Netzwerken thematisierte prominent Gomes-Casseres (1994). Vgl. ausführlicher Gomes-Casseres (1996) und außerdem Vanhaverbeke/Norderhaven (2001).

55 Vgl. Franz (2003); Schmid (2010).

56 Vgl. zum Beispiel im Biotechnologiesektor Powell/Koput/Smith-Doerr (1996), S. 142: „The locus of innovation is found within the networks of interorganizational relationships that sustain a fluid and evolving community." Vgl. auch Sydow/Möllering (2009), S. 257ff.

57 Vgl. früh zum Beispiel Perlmutter/Heenan (1986).

58 Vgl. hierzu im Einzelnen jüngst Wilhelm (2009). Bereits Gomes-Casseres (1994), S. 66, wies auf die Bedeutung des netzwerkinternen Wettbewerbs hin. Vgl. auch Baker/Faulkner/Fisher (1998). 
Das Phänomen des Netzwerkwettbewerbs ist nicht nur als Folge der „Marktverkrustung"59 vorstellbar. Auch neue Märkte, in denen noch Ungewissheit darüber besteht, ob sie sich überhaupt festigen, können dadurch stabilisiert werden, dass kooperativ vernetzte Unternehmen als Wettbewerber auftreten, wenn also etwa Kunden ihre Bedarfe bündeln oder Lieferanten ein gemeinsames Angebot für einen Auftrag abgeben. Auch können junge innovative Unternehmen in neuen Märkten durch Kooperation mit etablierten Unternehmen Zugang zu Vertriebskanälen und Kunden erhalten ${ }^{60}$. Dieses vor allem auf Skaleneffekte und das Erreichen einer kritischen Masse ausgelegte Vorgehen zur Reduzierung von Ungewissheit wird immer weniger nötig sein, je mehr der Markt und die Marktakteure wachsen. Doch lösen sich die Netzwerke nicht zwingend auf. Es kann ein direkter Übergang zu einem dauerhaften Netzwerkwettbewerb erfolgen ${ }^{61}$. Markteintritte sind dann noch über den Netzwerkeintritt möglich, soweit die Netzwerke - gerade wegen des internen und externen Wettbewerbs - flexibel und dynamisch bleiben ${ }^{62}$.

Ein Markt kann natürlich auch dadurch gekennzeichnet sein, dass nur ein Teil der Akteure kooperativ vernetzt ist und als Netzwerk konkurriert, während der andere Teil individuell auftritt, wie im klassischen Marktmodell. In dieser Konstellation sind zwei Ausprägungsformen von besonderem Interesse für die Marktentstehung. Zum einen ist es problematisch, wenn einige mächtige Unternehmen durch ihre Vernetzung die Marktteilnahme für kleine Unternehmen unmöglich machen, wenn sie also ihre marktbeherrschende Stellung als Netzwerk missbrauchen (ähnlich einem Kartell). Zum anderen bietet aber eine Vernetzung unter weniger mächtigen Akteuren eine Chance, den Wettbewerb mit mächtigen Oligopolisten oder gar Monopolisten erneut zu entfachen und den Markt funktionsfähig zu halten ${ }^{63}$. Im Stückgutmarkt etwa versetzen Kooperationen kleine und mittlere Speditionen in die Lage, mit den großen integrierten Logistikkonzernen zu konkurrieren ${ }^{64}$. So lässt sich festhalten, dass die Entstehung von Netzwerken die Marktentstehung fördert, wenn die Vernetzung den Wettbewerb im Markt belebt und die Netzwerke ein Mindestmaß an Offenheit wahren.

Einige Autoren weisen darauf hin, dass ein reiner Netzwerkwettbewerb höchst voraussetzungsvoll und damit empirisch selten ist, soweit damit die Notwendigkeit verbunden ist, dass Unternehmen sich exklusiv und dauerhaft einem einzigen Netzwerk anschließen ${ }^{65}$. Allerdings ist es im Hinblick auf Marktentstehung gerade interessant, dass Unternehmen mehrere Netzwerkmitgliedschaften (gegebenenfalls unterschiedlicher Intensität) haben können, es zu Ein- und Austritten kommt und somit in neuen Märkten

59 Lange (1998), S. 482. Es handelt sich um einen juristischen Begriff, der besagt, dass in einem Markt mehrere Netzwerke bestehen und jedes weitere Netzwerk vor diesem Hintergrund zu beurteilen ist.

60 Vgl. Bausch (2003), S. 195.

61 Auch hierfür sind Business Webs ein einschlägiges Beispiel, vgl. Schmid (2010).

62 Vgl. zum Beispiel Kröll (2003), S. 67ff., 195ff.; auch Sydow/Windeler/Wirth (2002).

63 Vgl. zu Kooperationen mittelständischer Unternehmen zum Beispiel Schmidt/Kiefer (2005), mit Blick auf internationale Kooperation auch Zentes/Swoboda (1999).

64 Vgl. Albers (2009), S. $124 \mathrm{f}$.

65 Vgl. mit Bezug speziell zur Automobilindustrie Rese (2006), S. 73; Wilhelm (2009), S. 48. 
auch ein Wettbewerb um Partner stattfindet (Bindungswettbewerb) ${ }^{66}$. Aus einer Managementperspektive ist daher die Möglichkeit der Marktgestaltung durch vielfältige Formen der Bindung an andere Marktakteure bedeutsam.

\section{Marktgestaltung durch Bindung: Organisierter Wettbewerb?}

Im Fokus dieses Beitrags steht wohlgemerkt nicht die Frage, wie Unternehmen durch die Kooperation mit anderen Unternehmen unter Berücksichtigung wettbewerbsrechtlicher Restriktionen ihre eigene Position in einem Markt optimieren können ${ }^{67}$, sondern wie die Zusammenarbeit das Entstehen des Marktes erst möglich macht: „complex forms of cooperation are usually necessary to promote competition"68. Wenn im Folgenden also ein bestimmter Bereich des unternehmerischen Handlungsraums in Marktentstehungsprozessen skizziert wird, so erinnert dies zwar an bekannte Analysen der Organisationsformen kooperativer Strategie aus dem Strategischen Management ${ }^{69}$, jedoch wird hier das Bewusstsein dafür geschärft, dass Bindungen zwischen Unternehmen Folgen auf der Marktebene haben ${ }^{70}$. Insoweit die Marktbedingungen rekursiv auf die Unternehmen zurückwirken, wird sodann ein reflexives Bindungsmanagement vorstellbar, das neue Märkte zu gestalten sucht und dabei zusehends in diese Märkte eingebettet wird. Die Marktakteure stehen dann nicht nur im Wettbewerb um Transaktionen, sondern eben auch im Wettbewerb um Bindungen.

\subsection{Bindungshorizont}

Kartelle, Konsortien und Kooperationen können einen Teil der Ungewissheit reduzieren, die einer Marktentstehung im Wege steht, und sie können den marktkonstitutiven Wettbewerb in Gang bringen, den sie auf den ersten Blick zu verhindern scheinen. Es gibt jedoch auch systematische Unterschiede zwischen diesen Formen der unternehmensübergreifenden Koordination, die in Abschnitt 2 bereits angedeutet wurden und hier auf zwei wesentliche Dimensionen reduziert werden: den Bindungshorizont und die Bindungsintensität (vgl. Abbildung 2) ${ }^{71}$. Der Bindungshorizont erfasst die zeitliche Perspektive der Koordination zwischen Marktakteuren. Es kommt dabei weniger auf die in Tagen, Monaten oder Jahren gemessene Bindungsdauer an, sondern darauf, ob

66 Vgl. Gimeno (2004), der das Verhältnis von Netzwerkwettbewerb zu Bindungswettbewerb auf das Maß an „alliance cospecialization“ (beziehungsspezifische Investitionen und Wissensbestände) zurückführt.

67 Auch bei Santos/Eisenhardt (2009), zum Beispiel S. 648, steht im Vordergrund, dass Unternehmen auch in entstehenden Märkten einem „monopolistic imperative“ folgen, den zum Beispiel auch schon Weber (1980[1922]), S. 382ff., beschrieb, der jedoch voraussetzt, dass sich der entsprechende Markt überhaupt etabliert.

68 Teece (1992), S. 3.

69 Vgl. u.a. Harrigan (1988); Bresser (1988; 1989); Osborn/Hagedoorn (1997); Jap (1999); Sjurts (2000); Bausch (2003); Albers (2005).

70 Vgl. zum Beispiel Phillips (1960); Hargrave/Van de Ven (2006).

71 Vgl. zu den nahezu unerschöpflichen Möglichkeiten und Varianten der Typologisierung von Kooperationen und Netzwerken: Sydow et al. (2003), S. 48-71. Bindungsdauer und -intensität sind u.a. zentral für die Unterscheidung zwischen stabilen und dynamischen Netzwerken bei Snow/Miles/Coleman (1992), vgl. Sydow et al. (2003), S. 86. 
die Akteure subjektiv empfinden, dass es sich um eine eher kurzfristige oder eher langfristige Bindung handelt. Für die Marktentstehung ist es bedeutsam, ob die sich zusammenschließenden Marktakteure von einer vorübergehenden oder dauerhaften Beschränkung des Wettbewerbs ausgehen. Je kürzer die Beschränkung (und je loser die Bindung, vgl. 3.2 unten), umso mehr erfolgt die Bindung im Lichte des Wettbewerbs.

\section{Abbildung 2: Bindungs- und Wettbewerbsformen}

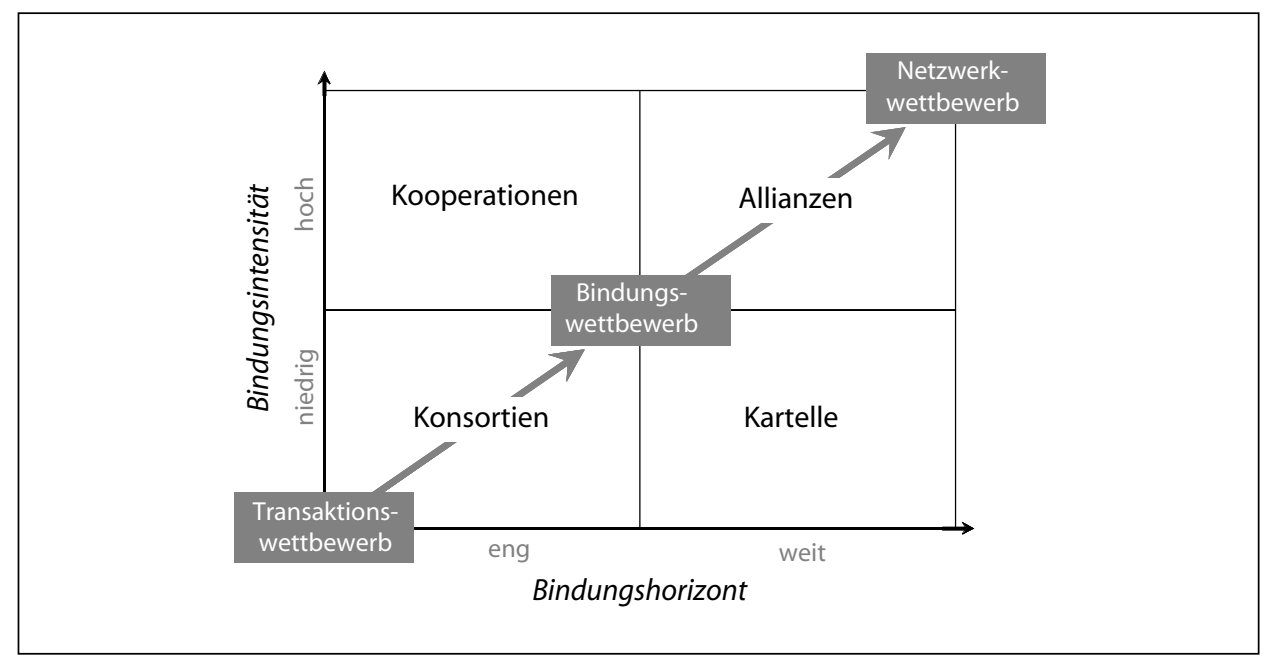

Erläuterung: Anhand der Dimensionen Bindungshorizont und -intensität lassen sich sowohl Bindungsformen (Kartell, Konsortium, Kooperation und Allianz) als auch Formen des Marktwettbewerbs (Transaktions-, Bindungs- und Netzwerkwettbewerb) unterscheiden.

Konsortien haben grundsätzlich einen engen Bindungshorizont, während die Absprachen in einem Kartell typischerweise bis auf Weiteres gelten und einen weiten Bindungshorizont eröffnen. In Konsortien sind sich die Konsorten sehr wohl bewusst, dass sie außerhalb des Konsortialgeschäfts Konkurrenten sind, also bereits zeitlich parallel zu der jeweils gerade gemeinsam unternommenen Aufgabe ${ }^{72}$. In Kartellen darf man sich hingegen zumindest einige Zeit unbehelligt fühlen, muss jedoch auch hier antizipieren, dass das Kartell aufgelöst oder gebrochen wird. Ein längerer Bindungshorizont bedeutet eine größere Reduzierung von Unsicherheit und könnte in frühen Phasen der Marktentstehung vorteilhaft sein. In späteren Phasen schadet er hingegen der Marktdynamik.

Kooperationen unterscheiden sich von Kartellen und Konsortien vor allem durch eine höhere Bindungsintensität (siehe unten). Dies bedeutet jedoch nicht, dass Kooperationen immer auch einen weiten Bindungshorizont haben (vgl. Abschnitt 2.4). Lang-

72 Vgl. auch Dowling/Lechner (1998). 
fristige Kooperationen werden hier als Strategische Allianzen bezeichnet. Wenn die Bindung eher kurzfristig angelegt (aber intensiv) ist, soll der Begriff der Kooperation verwendet werden. Entscheidend ist auch hier weniger, wie lange die Bindung dann tatsächlich Bestand hat, sondern vielmehr, ob die Partner bereits die mögliche Rückkehr in eine Wettbewerbsbeziehung zueinander antizipieren oder sich vorstellen, fortan dauerhaft als Allianz in Konkurrenz zu anderen Unternehmen und Allianzen zu stehen ${ }^{73}$. Mithin gehören unternehmerische Entscheidungen, ob und wann Bindungen gelöst werden, zu den zentralen strategisch-praktischen Ereignissen in der Marktentstehung.

\subsection{Bindungsintensität}

Sowohl aus der Sicht der Marktakteure als auch aus der Beobachterperspektive spielt neben dem Bindungshorizont die Intensität der Bindungen an andere Marktakteure in Kartellen, Konsortien und Kooperationen eine Rolle. Lose Bindungen einerseits und feste Bindungen andererseits bergen jeweils Chancen und Gefahren für die Beeinflussung der Marktentstehung, wobei man nicht pauschal sagen kann, dass eine geringe Bindungsintensität günstig und eine hohe Bindungsintensität ungünstig wäre, oder umgekehrt ${ }^{74}$. Um dies genauer betrachten zu können, braucht es zunächst eine Operationalisierung des Begriffes der Bindungsintensität. Diese kann man ermessen zum Beispiel anhand der von der Bindung zu einem Partner betroffenen Umsatzvolumina, Marktanteile, spezifischen Investitionen, Informationsflüsse, Anzahl (Multiplexität) und strategischen Bedeutung der Geschäftsbereiche, bis hin zum Ausmaß der gegenseitigen Kapitalbeteiligungen. Die Intensität der Bindung zu einem Partner beruht auf formalen Festlegungen in Verträgen, aber auch auf Gewohnheiten und impliziten Annahmen sowie subjektiven Wahrnehmungen. Während aus der Sicht einer Unternehmung zunächst die Bindungsintensität zu jedem einzelnen seiner Partner im Vordergrund stehen mag, spielt außerdem das Beziehungsgeflecht zwischen mehreren Partnern eine Rolle. Zieht man auf der Netzwerkebene gängige Koeffizienten wie die Netzwerkdichte heran und verknüpft sie etwa mit den dyadischen Beziehungsintensitäten, so kann man auch Aussagen über die Beziehungsintensität eines Netzwerks machen ${ }^{75}$.

Entscheidungen der Marktakteure darüber, ob und wie stark sie Bindungen intensivieren, wirken sich auf den Wettbewerb in einem entstehenden Markt aus. Enge Bindungen reduzieren zwar zunächst die Konkurrenz zwischen den Partnern (einmal abgesehen von möglichen Verteilungskonflikten) ${ }^{76}$, können jedoch einen ausgeprägten Netzwerkwettbewerb zwischen Allianzen befördern. Weniger intensive Bindungen bis hin zu lediglich latenten Beziehungen entsprechen eher dem atomistischen Bild des klassischen Wettbewerbsmarktes, können aber dennoch nachhaltig strukturierend

73 Vgl. die Vorstellung von „group versus group“ bei Gomes-Casseres (1994).

74 Zum Begriff der Bindungsintensität vgl. insbes. Schubert/Küting (1981), S. 8f. Zum Teil wird von der Schärfe oder Strenge der Bindung gesprochen. In der Wirtschaftssoziologie wird mit Granovetter $(1973 ; 1985)$ zwischen „strong ties" und „weak ties“ unterschieden, welche die Einbettung von Akteuren in einen Markt ausmachen und die je eigene Vor- und Nachteile bergen. Vgl. auch Uzzi (1997).

75 Vgl. zum Beispiel Wasserman/Faust (1994). Für Business Webs vgl. Schmid (2010).

76 Vgl. hierzu etwa Semlinger (1993), S. 337ff., oder Schmidtchen (2005), S. 67ff. 
wirken und zum Beispiel die Herausbildung von Marktsegmenten zur Folge haben, indem Marktakteure den Kreis möglicher Transaktions- und Kooperationspartner auf Bekannte beschränken ${ }^{77}$. Sehr deutlich kommt die Strukturierung möglicher Transaktionspartner etwa in der Rede von „In-Suppliers“ im Gegensatz zu „Out-Suppliers“ zum Ausdruck ${ }^{78}$, der in der Beschaffungspraxis heute der sogenannte Preferred-Supplier-Status entspricht ${ }^{79}$. Die Bindung muss dabei nicht einmal unbedingt zu einzelnen Akteuren bestehen. Auch der Status der Marktakteure kann als Mechanismus der Etablierung und Ordnung von Märkten dienen ${ }^{80}$. Und auch der technologische "Lock-in“ als typischer Effekt von Business Webs ${ }^{81}$ prägt Marktstrukturen.

Es ist im Übrigen durchaus möglich (und effizient), dass Unternehmen gleichzeitig engere und losere Bindungen in ihren Netzwerken pflegen ${ }^{82}$. Gerade in der Phase der Marktentstehung, in der die Legitimität der Marktakteure und des Marktes noch nicht gesichert ist, brauchen Unternehmen sowohl enge Verbündete und Unterstützer als auch die nötige Offenheit für neue Kontakte ${ }^{83}$. Die Frage, wie intensiv man kooperiert, ist dann auch gekoppelt an die Frage, wie lange die Bindung dauern soll. Wohlgemerkt haben individuelle Entscheidungen der Unternehmen über ihre Bindungsportfolios Auswirkungen auf die Entstehung und weitere Entwicklung des Marktes insgesamt.

\subsection{Bindungsdynamik}

Die in den vorigen Abschnitten und in Abbildung 2 zusammengefassten Bindungsformen mit jeweils engem oder weitem Bindungshorizont und hoher oder niedriger Bindungsintensität unterliegen einer gewissen Dynamik, zumal in neuen Märkten. Mit Bindungsdynamik ist gemeint, dass jede Bindung die Voraussetzungen für ihre eigene Weiterentwicklung sowie die Entwicklung von Netzwerken und Märkten schafft. So gibt es die Neigung zu Transaktionen und Kooperationen mit bereits Bekannten ${ }^{84}$ oder eine tendenziell längere Dauer von intensiven Bindungen. Auch in der Analyse der Dynamik von Marktentstehungsprozessen ist zu beachten, dass (Kooperations-)Entscheidungen nicht vollständig rückgängig gemacht werden können, sondern zukünftige Bindungen vorstrukturieren und gar Pfadabhängigkeiten schaffen können ${ }^{85}$.

Unter dem Aspekt der Dynamik kann zunächst jede der vier hier explizierten Bindungsformen für sich betrachtet werden. Kartelle, Konsortien, Kooperationen und Allianzen durchlaufen, vereinfachend dargestellt, Phasen der Anbahnung, Aushandlung, Ausführung und Auflösung, deren genaue Ausprägung und Abfolge mit der Bindungs-

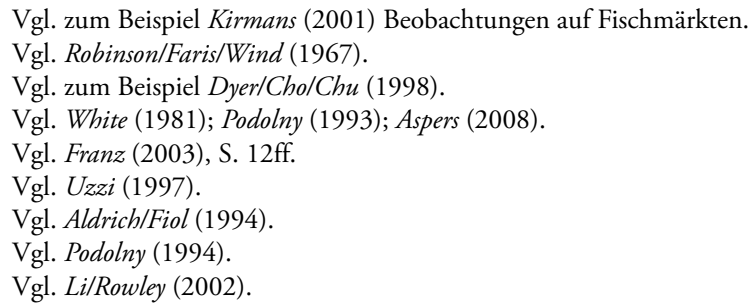


form zusammenhängt. Des Weiteren stellt sich die für den Wettbewerb in einem neuen Markt sehr bedeutende Frage, ob Bindungen mit der Zeit zur Intensivierung und zeitlichen Ausdehnung neigen, es mithin eine Dynamik geben könnte, wonach zum Beispiel aus relativ losen Konsortien dann intensivere Kooperationen bis hin zu Allianzen erwachsen: „Initial collaborations begin informally, in networks of interfirm relations, but some later develop into more formalized strategic alliances, consortia, and trade

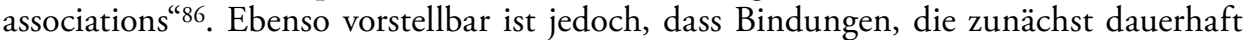
und intensiv angelegt waren, im Zuge der Marktentwicklung zusehends aufgelöst und durch flexiblere Bindungsformen ersetzt werden ${ }^{87}$. Der Wettbewerb hängt also nicht nur von Bindungen ab, sondern Bindungen werden auch vor dem Hintergrund des jeweiligen Wettbewerbs eingegangen oder eben gelöst ${ }^{88}$. Kim/Oh/Swaminathan prognostizieren: „The more competitive the environment, the more likely an organization is to change its network ties" 89 .

Einmal mehr ist es von Bedeutung, dass Wettbewerb zwischen Marktakteuren und auch zwischen Netzwerken (Gruppen verbundener Akteure) stattfinden kann (siehe 2.5 oben). So kann man nicht pauschal sagen, dass mehr Bindungen zu weniger Wettbewerb führen oder dass steigender Wettbewerb Bindungen fördert oder zerstört, sondern muss genauer analysieren, ob und wie die Marktakteure ihre Teilnahme am Wettbewerb über Bindungen gestalten und ob sie dabei von einer Bindungsform zu einer anderen übergehen. Auf der Marktebene betrachtet ist außerdem folgende Dynamik interessant: Führt beispielsweise die Gründung einer Allianz zu weiteren Allianzen unter anderen Unternehmen als Gegenreaktion (zum Beispiel Luftverkehr) ${ }^{90}$ ? Oder wird etwa die Abwicklung von Geschäften über ein Konsortium zu einem Modell, das viele Unternehmen in einem Markt imitieren (zum Beispiel Emissionen im Bankwesen) ${ }^{91}$ ? Individuelle Kooperationsentscheidungen wie auch etwa eine systematische (zum Beispiel staatliche) Kooperationsförderung in neuen Märkten können so zur dynamischen Ausbreitung von Bindungsformen führen und in ein dominantes und für den jeweiligen Markt typisches Bindungs- und Wettbewerbsmuster münden, wie zum Beispiel FastFood-Franchising ${ }^{92}$, Projektnetzwerke in der Produktion von Fernsehsendungen ${ }^{93}$, Business Webs im Bereich Internetfernsehen ${ }^{94}$ oder auch Systempartnerschaften zwischen Herstellern und Lieferanten in der Automobilindustrie95. 


\subsection{Bindungsmanagement}

Marktakteure gehen Bindungen zu anderen Akteuren bewusst ein. Zwar haben die Akteure vor allem in der Entstehungsphase eines neuen Marktes nur bedingt die Kontrolle über die Umstände und Folgen ihrer Bindungen, müssen auf Zufälle und Unvorhergesehenes reagieren und sehen ihre früheren Entscheidungen später in einem anderen Licht. Dennoch versuchen sie zumindest, ihre Bindungen vorteilhaft zu gestalten ${ }^{96}$. Ein reflexives Bindungsmanagement lernt aus der Vergangenheit und plant für die $\mathrm{Zu}-$ kunft, trotz aller Unwägbarkeiten ${ }^{97}$. Die oben angesprochene Bindungsdynamik ist sowohl das Resultat von Entscheidungen der Marktakteure als auch - und zwar rekursiv verknüpft - der Rückkopplungen aus den Netzwerken und Märkten ${ }^{98}$.

Bindungsmanagement ist gängige Praxis, wenn man etwa an Lieferantenentwicklungs-, Kundenbindungs- oder Vernetzungsprogramme denkt. Geschäftsbeziehungen anbahnen und pflegen zu müssen, ist in realen Märkten geradezu eine kaufmännische Selbstverständlichkeit ${ }^{99}$. Weniger verbreitet ist allerdings ein strategisches Bindungsmanagement, bei dem Unternehmen über ihr eigenes Bindungsportfolio hinaus die Strukturen des (neuen) Marktes im Blick haben ${ }^{100}$. So könnten etwa zwei Unternehmen auf eine Allianz verzichten, obwohl sie zunächst ökonomisch sinnvoll und wettbewerbsrechtlich unproblematisch erscheint, weil sie antizipieren, dass ihre Wettbewerber als Reaktion ebenfalls Allianzen eingehen würden und der Markt damit insgesamt weniger attraktiv werden könnte ${ }^{101}$. Ebenso gehen nur wenige Unternehmen so weit, die „Architektur“ ihrer Beschaffungsmärkte zu beeinflussen, indem sie etwa neue Lieferanten fördern, um den Konkurrenzdruck auf vorhandene Lieferanten zu erhöhen. Absatzseitig ist für Marktakteure wichtig, dass sie über ihre bestehenden Distributionskanäle hinausblicken und zum Beispiel im Interesse des Gesamtmarktes reagieren, wenn einzelne Händler zu dominant werden. Die Beeinflussung der Beschaffungs- und Absatzmärkte kann also vor allem über das Bindungsmanagement erfolgen ${ }^{102}$.

Da Kartelle, Konsortien und Kooperationen die Entstehung neuer Märkte fördern - aber auch verhindern - können, kommt dem Bindungsmanagement in der Marktentstehungsphase eine besondere Bedeutung zu. Unternehmen gehen Bindungen ein, um sich selbst als Marktakteure sowie die anderen Elemente der Marktkonstitution zu erschaffen, insbesondere Produkte, Standards und Regeln für den neuen Markt. Sie sollten dabei wohlgemerkt nicht nur ihre eigenen, konkreten Lieferanten und Kunden

96 Vgl. Steiner (2004). Auf Business Webs bezogen spricht Franz (2003) von „Partnermanagement“.

97 Vgl. Sydow (2001).

98 Vgl. Windeler (2001), S. 200ff.

99 Allgemeine zusätzliche Funktionen des Netzwerkmanagements wurden insbes. von Sydow/Windeler (1994) herausgestellt, bedeuten aber für die Praxis nach wie vor eine Herausforderung, insbesondere die Herausbildung der von Hoffmann (2003) untersuchten „Allianzmanagementkompetenz“; dazu unter dem Stichwort „organisationale Beziehungsfähigkeiten“ auch Pfohl/Buse (1999).

100 Vgl. den Ansatz von Aldrich/Fiol (1994), die jedoch vor allem auf die Legitimität der Aktivitäten neuer Unternehmen auf neuen Märkten fokussieren.

101 Vgl. hierzu auch Gimeno (2004).

102 Vgl. zu strategischen Fragen der „Vertical Architecture“ Jacobides/Billinger (2006) und Jacobides/Knudsen/Augier (2006) sowie insbes. zur Marktentstehung durch Disintegration Jacobides (2005). 
im Blick haben, sondern die breitere Lieferanten- und Kundenbasis, die den Markt insgesamt trägt. Deshalb engagieren sich Marktakteure auch in Verbänden und tragen freiwillig zu Kollektivgütern bei, müssen diese jedoch in der Marktentstehungsphase erst noch gemeinsam aufbauen ${ }^{103}$. Da viele Unternehmen zuerst nur ihre eigenen Bindungen managen mögen, ist es umso wichtiger, dass es auch Akteure gibt, die als Regulatoren, Intermediäre oder "Marktführer" (im doppelten Wortsinn) den Überblick über die Marktstrukturierung durch Bindungen behalten ${ }^{104}$. Aus der Sicht der Marktakteure dient Bindungsmanagement mithin dem doppelten Ziel der Ermöglichung des Marktes insgesamt und der Erlangung einer möglichst vorteilhaften Position in ihm, wobei einzelne Maßnahmen oft nicht beiden Zielen gleichermaßen dienlich sind und die Gefahr entweder markt- oder geschäftsschädigenden Verhaltens besteht.

\subsection{Bindungswettbewerb}

Der Wettbewerb in neuen Märkten wird also nicht nur zwischen Unternehmen, sondern auch zwischen Unternehmensnetzwerken aufgebaut und ausgetragen (Netzwerkwettbewerb, vgl. Abschnitt 2.5 oben). Das Bindungsmanagement kann daher als wettbewerbsförderlich betrachtet werden, obschon jede Bindung als solche erst einmal Wettbewerb durch Kooperation ersetzt ${ }^{105}$. Wie bereits angesprochen wurde, gibt es jedoch auch in Netzwerken Konkurrenz um die Aufnahme und den Verbleib in einem starken Verbund und um die Erlangung einer attraktiven Position in ihm (gemessen zum Beispiel an Status, Reputation, Ressourcenzugang, strategischer Führerschaft etc.). Neue Mitglieder werden sorgfältig ausgesucht und bereits aufgenommene Mitglieder müssen sich hin und wieder dem „Markttest“ unterwerfen, das heißt: ihre Leistungsfähigkeit beweisen und sich mit Alternativkandidaten messen lassen ${ }^{106}$.

Neben den Wettbewerb zwischen Netzwerken tritt also der Wettbewerb um die Einbindung in attraktive Netzwerke ${ }^{107}$. Dieser Bindungswettbewerb bringt Dynamik in einen neuen Markt, in dem der Wettbewerb um Transaktionen durch kooperative Beziehungen reduziert sein mag. Vor allem in der Analyse von neuen Märkten, aber auch darüber hinaus, sollte man also einen weiten Wettbewerbsbegriff verwenden, der nicht nur die klassische Konkurrenz zwischen Marktakteuren um einzelne Transaktionen, sondern auch den Wettbewerb zwischen Netzwerken und insbesondere auch den Wettbewerb um Bindungen zu anderen Marktakteuren einschließt. Unternehmen müssen entscheiden, ob sie allein oder kollektiv am Markt auftreten, und sie müssen im letzteren Fall attraktive Partner finden und für diese attraktiv sein. Ob eine Bindung die gemeinsame Position am Markt verbessert und gar „kooperative Kernkompetenzen“108

103 Vgl. Phillips (1960); Aldrich/Fiol (1994).

104 Vgl. noch einmal Phillips (1960), S. 608f., sowie Aldrich/Fiol (1994), S. 655, 658, die von „industry champions“ sprechen, die zum Beispiel die Gründung von Verbänden vorantreiben.

105 Verbindungen zwischen Marktakteuren „dämpfen“ (Zohlnhöfer (1991), S. 80) die Wettbewerbsintensität, dies mitunter jedoch auf ein Maß, das erst einen funktionsfähigen Wettbewerb gestattet.

106 Vgl. Jarillo (1988), S. 35, sowie u.a. Snow/Miles/Coleman (1992); Wilhelm (2009).

107 Hargrave/Van de Ven (2006), S. 873: „Collaboration may itself become a dimension of competition.“

108 Duschek (1998), vgl. Dyer/Singh (1998). 
entstehen, ist zudem nicht im Voraus garantiert, sondern stellt sich oft erst im Laufe der Zusammenarbeit heraus, wenn die Partner Ressourcen kombinieren und Routinen etablieren. Bleibt der Erfolg aus, dann werden Bindungen gelöst und neue Partner gesucht. Die entstehenden dynamischen Bindungsmuster tragen sodann den Markt als Transaktionssystem.

\section{Fazit und Forschungsperspektiven: Kooperation ermöglicht Konkurrenz}

Wenn Unternehmen sich in Kartellen, Konsortien oder anderen Formen der Kooperation engagieren, so wird gemeinhin angenommen, dass dies den Wettbewerb einschränkt und dass damit das Entstehen oder auch das Fortbestehen eines Marktes gefährdet wird. Dies ist, mit David Teece gesprochen, der Schatten, den das neoklassische Denken noch immer auf die Wettbewerbspolitik wirft ${ }^{109}$. In diesem Beitrag wurde hingegen argumentiert, dass Kooperation Konkurrenz ermöglicht und in der Marktentstehung eine wichtige Rolle spielt. Man kann sogar so weit gehen und das Verkümmern eines neuen Marktes nicht auf mangelnden Wettbewerb, sondern auf mangelnde Kooperation zwischen Marktakteuren zurückführen ${ }^{110}$. Welche Wirkung Kooperation auf Märkte hat, hängt davon ab, ob sie als Mittel im Wettbewerb eingesetzt wird oder eben doch, wie klassisch vermutet, zur Blockierung von Wettbewerb. Die Vorstellung von Kooperation als Mittel im Wettbewerb wiederum erfordert einen erweiterten Begriff des Marktwettbewerbs. Unternehmen kooperieren, damit sie dann alleine stark genug werden, um erfolgreich als Anbieter oder Nachfrager auftreten zu können (Transaktionswettbewerb); sie konkurrieren im Verbund mit ihren Partnern gegen andere kollektive Marktakteure (Netzwerkwettbewerb); und sie müssen sich die Gunst ihrer aktuellen und zukünftigen Partner erstreiten (Bindungswettbewerb). Ein reflexives Bindungsmanagement dient der dauerhaften Etablierung und Reproduktion eines neuen Marktes und nicht nur der kurzfristigen Optimierung der individuellen Marktposition. In der Marktentstehungsphase wird deutlich, dass der Markt als Kollektivgut entwickelt und gepflegt werden muss.

Eine empirische Forschung, die diesen neuen Blick auf die kreativen Wirkungen von Kooperation und Konkurrenz in der Marktentstehung verfolgt, kann die in diesem Beitrag skizzierten Kooperations- und Wettbewerbsformen analytisch nutzen (vgl. $A b$ bildung 2 oben). Im Vergleich zu anderen Typologien stellt der hier anhand der Dimensionen Bindungshorizont und Bindungsintensität aufgespannte Analyseraum stark auf das Maß der Marktlichkeit der Beziehungen zwischen den Marktakteuren ab, wobei das neoklassische Ideal der punktuellen Transaktion im Nullpunkt liegt, von dem reale Marktbeziehungen mehr oder weniger weit entfernt liegen.

109 Vgl. Teece (1992), S. 23.

110 Vgl. auch Aldrich/Fiol (1994), S. 665, die zur Untersuchung von gescheiterten Fällen aufrufen, um so die Faktoren für eine erfolgreiche Etablierung zu finden bzw. zu bestätigen. 
Hierauf aufbauend kann empirisch untersucht werden, welche Beziehungsformen Unternehmen in der Marktentstehungsphase wählen. In einem etablierten Markt, in dem der Transaktionswettbewerb im Vordergrund steht, wäre zu erwarten, dass mit weiter zunehmender Reife kollektive Strategien gewählt werden, sich ein entsprechender Bindungswettbewerb um attraktive Partner entwickelt, der dann bei zunehmender Verfestigung von Partnerschaften zu einem Netzwerkwettbewerb führt, das heißt: zu einem Transaktionswettbewerb zwischen Netzwerken. In der Phase der Marktentstehung ist jedoch eine umgekehrte Abfolge denkbar: Bevor ein Transaktionswettbewerb möglich ist - und sich damit der neue Markt zu etablieren beginnt - arbeiten die potentiellen Marktakteure mit festen Partnern, mit denen sie die ersten Investitionen tätigen, ein eigenes Profil entwickeln und die konstitutiven Elemente des neuen Marktes herausarbeiten. Wenn dann Transaktionen stattfinden, verstärkt sich auch der Wettbewerb innerhalb der Kooperationen. Bindungen verlangen Bestätigung („Markttest“"111) oder sie werden gelöst und neue Partner gesucht (Bindungswettbewerb). Wenn also von vorwettbewerblichen Aktivitäten in der Marktentstehung die Rede ist, so bezieht sich dies auf den noch nicht stattfindenden Transaktionswettbewerb im engeren Sinne, was jedoch keineswegs bedeutet, dass die entsprechenden Unternehmen nicht bereits um günstige Positionen und Partner konkurrieren ${ }^{112}$. Zukünftige Forschungen können diese Dynamik der Bindungshorizonte und -intensitäten in neuen Märkten empirisch erfassen.

Konzeptionell bereitet dieser Beitrag den Boden für eine Erweiterung der Forschung in Strategischem Management und Marketing. Die bisherigen Erkenntnisse zu kollektiven und kooperativen Strategien in etablierten Märkten werden nicht bezweifelt, sondern es wird das zusätzliche Potenzial identifiziert, das sich einem Management bietet, welches sich schon früh in unternehmensübergreifenden Prozessen der Marktentstehung engagiert $^{113}$. Auch für die Wettbewerbstheorie sowie wettbewerbspolitische Abwägungen wird eine Erweiterung des Horizonts über den Transaktionswettbewerb hinaus angeregt, nicht etwa die Verneinung der zentralen Rolle des Wettbewerbs im Allgemeinen.

$\mathrm{Zu}$ den immer wieder gern angebrachten Zitaten von Adam Smith gehört neben der Beschreibung der unsichtbaren Hand des Marktes und der zentralen Feststellung, dass der (marktgemäße) Handel in der Natur des Menschen liegt, auch die Behauptung, dass Marktakteure zu konspirativen (markthinderlichen) Absprachen neigen ${ }^{114}$. Der Widerspruch löst sich auf, wenn nicht der Markt als ein natürliches Prinzip erachtet wird, sondern reale Märkte als kollektiv zu konstituierende Transaktionssysteme zwischen Kooperation und Konkurrenz erkannt werden. Während Smith also zum Beispiel gegen öffentliche Handelsregister argumentierte ${ }^{115}$, wären solche Register nach der hier vertretenen Auffassung ein Mittel der Markterschaffung. Die schon von Almarin Phillips hervorgehobene Gruppenorientierung von Unternehmen in einem Markt, die neben individuellen Gewinnmaximierungszielen besteht, ist auf neu entstehenden Märkten

111 Jarillo (1988), S. 35.

112 Vgl. zum Beispiel Geroski (2003), S. 97.

113 Vgl. Humphreys (2010).

114 Vgl. Smith (1976[1776]), S. 25ff. und 145.

115 Vgl. Smith (1976[1776]), S. 145, der in Registern ein Hilfsmittel zur Kollusion sieht. 
höchst relevant ${ }^{116}$. Vor allem durch verschiedene Formen der Kooperation tragen Unternehmen dazu bei, das Spiel zu erschaffen, in dem sie dann später gut positioniert und erfolgreich sein wollen. Chancen werden verpasst, wenn man erwartet, dass neue Spiele von selbst entstehen, oder man erst einsteigt, wenn das Spiel längst begonnen hat.

\section{Literatur}

Albers, Sascha (2005), The Design of Alliance Governance Systems, Köln.

Albers, Sascha (2009), Kooperationsorganisation von Stückgutnetzwerken, in: Albers, Sascha/Reihlen, Markus (Hrsg.), Management integrierter Wertschöpfungsnetzwerke, Köln, S. 113-144.

Aldrich, Howard E./Fiol, C. Marlene (1994), The institutional context of industry creation, in: Academy of Management Review, Vol. 19, S. 645-670.

Anderson, Erin/Gatignon, Hubert (2005), Firms and the creation of new markets, in: Menard, Claude/Shirley, Mary M. (Hrsg.), Handbook of New Institutional Economics, Dordrecht, S. 401-431.

Appleyard, Melissa M./Wang, Clara Y./Liddle, J. Alexander/Carruthers, John (2008), The innovator's non-dilemma: The case of next-generation lithography, in: Managerial \& Decision Economics, Vol. 29, S. 407-423.

Aspers, Patrik (2008), Order in garment markets, in: Acta Sociologica, Vol. 51, S. 187-202.

Baker, Wayne E./Faulkner, Robert R./Fisher, Gene A. (1998), Hazards of the market: The continuity and dissolution of interorganizational market relationships, in: American Sociological Review, Vol. 63, S. 147-177.

Bausch, Andreas (2003), Unternehmungszusammenschlüsse: Strategien und Strukturen für kooperatives und akquisitorisches Wachstum in Industrieunternehmungen, Wiesbaden.

Biggart, Nicole W./Delbridge, Rick (2004), Systems of exchange, in: Academy of Management Review, Vol. 29, S. 28-49.

Bradach, Jeffrey L. (1997), Using the plural form in the management of restaurant chains, in: Administrative Science Quarterly, Vol. 42, S. 276-303.

Bresser, Rudi K. F. (1988), Matching collective and competitive strategies, in: Strategic Management Journal, Vol. 9, S. 375-385.

Bresser, Rudi K. F. (1989), Kollektive Unternehmensstrategien, in: Zeitschrift für Betriebswirtschaft, 59. Jg., S. $545-$ 564.

Bresser, Rudi K. F./Harl, Johannes E. (1986), Collective strategy: Vice or virtue?, in: Academy of Management Review, Vol. 11, S. 408-427.

Callon, Michel (1998), Introduction: The embeddedness of economic markets in economics, in: Callon, Michel (Hrsg.), The Laws of the Markets, Oxford, S. 1-68.

Conrad, Christian A. (2005), Co-opetition und funktionsfähiger Wettbewerb, in: Wirtschaftswissenschaftliches Studium, 34. Jg., S. 169-171.

Cook, Karen S. (1977), Exchange and power in networks of interorganizational relations, in: Sociological Quarterly, Vol. 18, S. 62-82.

Corsten, Hans (2003), Zwischen Aufgabe und Zuwachs an Autonomie - Unternehmungsnetzwerke, in: Küting, Karlheinz/Noack, Hans-Christoph (Hrsg.), Der große BWL-Führer: Die 50 wichtigsten Strategien und Instrumente zur Unternehmensführung, Frankfurt/M., S. 54-61.

Cyert, Richard M./March, James G. (1963), A Behavioral Theory of the Firm, Englewood Cliffs.

Das, T. K./Teng, Bing-Sheng (2000), Instabilities of strategic alliances: An internal tensions perspective, in: Organization Science, Vol. 11, S. 77-101.

116 Vgl. Phillips (1960). 


\section{G. MÖLLERING}

Dickson, Pat H./Weaver, K. Mark (1997), Environmental determinants and individual-level moderators of alliance use, in: Academy of Management Journal, Vol. 40, S. 404-425.

Dowling, Michael/Lechner, Christian (1998), Kooperative Wettbewerbsbeziehungen: Theoretische Ansätze und Managementstrategien, in: Die Betriebswirtschaft, 58. Jg., S. 86-102.

Duschek, Stephan (1998), Kooperative Kernkompetenzen - Zum Management einzigartiger Netzwerkressourcen, in: Zeitschrift Führung + Organisation, 67. Jg., S. 230-236.

Dyer, Jeffrey H./Cho, Dong S./Chu, Wujin (1998), Strategic supplier segmentation: The next „Best Practice“ in supply chain management, in: California Management Review, Vol. 40, S. 57-77.

Dyer, Jeffrey H./Singh, Harbir (1998), The relational view: Cooperative strategy and sources of interorganizational competitive advantage, in: Academy of Management Review, Vol. 23, S. 660-679.

Engelhard, Johann/Sinz Elmar (Hrsg.) (1999), Kooperation im Wettbewerb: Neue Formen und Gestaltungskonzepte im Zeichen von Globalisierung und Informationstechnologie, Wiesbaden.

Fligstein, Neil (1990), The Transformation of Corporate Control, Cambridge/MA.

Fligstein, Neil (2001), The Architecture of Markets, Princeton.

Franz, Andreas (2003), Management von Business Webs: Das Beispiel von Technologieplattformen für mobile Dienste, Wiesbaden.

Gawer, Annabelle (2009), Platforms, Markets and Innovation, Cheltenham.

Geroski, Paul A. (2003), The Evolution of New Markets, Oxford.

Gimeno, Javier (2004), Competition within and between networks: The contingent effect of competitive embeddedness on alliance formation, in: Academy of Management Journal, Vol. 47, S. 820-842.

Gomes-Casseres, Benjamin (1994), Group versus group: How alliance networks compete, in: Harvard Business Review, Vol. 72, S. 62-74.

Gomes-Casseres, Benjamin (1996), The Alliance Revolution: The New Shape of Business Rivalry, Cambridge/MA.

Granovetter, Mark (1973), The strength of weak ties, in: American Journal of Sociology, Vol. 78, S. 1360-1380.

Granovetter, Mark (1985), Economic action and social structure: The problem of embeddedness, in: American Journal of Sociology, Vol. 91, S. 481-510.

Hagedoorn, John/Frankort, Hans T. W. (2008), The gloomy side of embeddedness: The effects of overembeddedness on inter-firm partnership formation, in: Baum, Joel A. C./Rowley, Timothy J. (Hrsg.), Advances in Strategic Management, Vol. 25: Network Strategy, Bingley, S. 503-530.

Hamel, Gary (1991), Competition for competence and inter-partner learning within international strategic alliances, in: Strategic Management Journal, Vol. 12, S. 83-103.

Hargrave, Timothy J./Van de Ven, Andrew (2006), A collective action model of institutional innovation, in: Academy of Management Review, Vol. 31, S. 864-888.

Harrigan, Kathryn R. (1988), Joint ventures and competitive strategy, in: Strategic Management Journal, Vol. 9, S. 141-158.

Hayek, Friedrich A. (1973), Law, Legislation and Liberty: A New Statement of the Liberal Principles of Justice and Political Economy, Vol. 1: Rules and Order, Chicago.

Hoffmann, Werner H. (2003), Allianzmanagementkompetenz - Entwicklung und Institutionalisierung einer strategischen Ressource, in: Schreyögg, Georg/Sydow, Jörg (Hrsg.), Managementforschung 13: Strategische Prozesse und Pfade, Wiesbaden, S. 93-150.

Humphreys, Ashlee (2010), Megamarketing: The creation of markets as a social process, in: Journal of Marketing, Vol. 74, S. 1-19.

Ingram, Paul/Inman, Crist (1996), Institutions, intergroup competition, and the evolution of hotel populations around Niagara Falls, in: Administrative Science Quarterly, Vol. 41, S. 629-658.

Jacobides, Michael G. (2005), Industry change through vertical disintegration: How and why markets emerged in mortgage banking, in: Academy of Management Journal, Vol. 48, S. 465-498. 
Jacobides, Michael G./Billinger, Stephan (2006), Designing the boundaries of the firm: From „Make, Buy, or Ally“ to the dynamic benefits of vertical architecture, in: Organization Science, Vol. 17, S. 249-261.

Jacobides, Michael G./Knudsen, Thorbjorn/Augier, Mie (2006), Benefiting from innovation: Value creation, value appropriation and the role of industry architectures, in: Research Policy, Vol. 35, S. 1200-1221.

Jap, Sandy D. (1999), Pie-expansion efforts: Collaboration processes in buyer-supplier relationships, in: Journal of Marketing Research, Vol. 36, S. 461-475.

Jarillo, J. Carlos (1988), On strategic networks, in: Strategic Management Journal, Vol. 9, S. 31-41.

Kim, Tai-Young/Oh, Hongseok/Swaminathan, Anand (2006), Framing interorganizational network change: A network inertia perspective, in: Academy of Management Review, Vol. 31, S. 704-720.

Kirchner, Christian (1999), Rechtliche Rahmenbedingungen für Kooperationen: Eine kritische Analyse des deutschen und europäischen Wettbewerbs- und Kartellrechts, in: Engelhard, Johann/Sinz, Elmar (Hrsg.), Kooperation im Wettbewerb: Neue Formen und Gestaltungskonzepte im Zeichen von Globalisierung und Informationstechnologie, Wiesbaden, S. 445-477.

Kirman, Alan (2001), Market organization and individual behaviour: Evidence from fish markets, in: Rauch, James E./Casella, Alessandra (Hrsg.), Networks and Markets, New York, S. 155-195.

Knight, Frank H. (1971[1921]), Risk, Uncertainty, and Profit, Chicago.

Kogut, Bruce (1988), Joint ventures: Theoretical and empirical perspectives, in: Strategic Management Journal, Vol. 9, S. 319-332.

Kogut, Bruce (1989), The stability of joint ventures: Reciprocity and competitive rivalry, in: Journal of Industrial Economics, Vol. 38, S. S. 183-198.

Kröll, Anna-Martina (2003), Interorganisationale Netzwerke - Nutzung Sozialen Kapitals für Markteintrittsstrategien, Wiesbaden.

Lange, Knut W. (1998), Das Recht der Netzwerke: Moderne Formen der Zusammenarbeit in Produktion und Vertrieb, Heidelberg.

Lange, Knut W. (2001), Rechliche Probleme Virtueller Netzwerke, in: Corsten, Hans (Hrsg.), Unternehmungsnetzwerke: Formen unternehmungsübergreifender Zusammenarbeit, München, S. 271-298.

Li, Stan X./Rowley, Timothy J. (2002), Inertia and evaluation mechanisms in interorganizational partner selection: Syndicate formation among U.S. investment banks, in: Academy of Management Journal, Vol. 45, S. 11041119.

Lieberman, Marvin B./Montgomery, David B. (1988), First-mover advantages, in: Strategic Management Journal, Vol. 9, S. 41-58.

Möllering, Guido (2009a), Market constitution analysis: A new framework applied to solar power technology markets, MPIfG Working Paper 09/7, Köln.

Möllering, Guido (2009b), Konsortien: Eine altbekannte Kooperationsform mit Forschungsbedarf, in: Wirtschaftswissenschaftliches Studium, 38. Jg., S. 591-593.

North, Douglass C. (1990), Institutions, Institutional Change and Economic Performance, Cambridge.

Oliver, Christine (1990), Determinants of interorganizational relationships: Integration and future directions, in: Academy of Management Review, Vol. 15, S. 241-265.

Ortmann, Günther (2005), Das fatale Apriori des Marktes. Kommentar zum Beitrag von Jochen Koch, in: Schauenberg, Bernd/Schreyögg, Georg/Sydow, Jörg (Hrsg.), Managementforschung 15: Institutionenökonomik als Managementlehre?, Wiesbaden, S. 229-237.

Ortmann, Günther/Zimmer, Marco (1998), Strategisches Management, Recht und Politik, in: Die Betriebswirtschaft, 58. Jg., S. 747-769.

Osborn, Richard N./Hagedoorn, John (1997), The institutionalization and evolutionary dynamics of interorganizational alliances and networks, in: Academy of Management Journal, Vol. 40, S. 261-278. 


\section{G. MÖLLERING}

Ostrom, Elinor (2005), Doing institutional analysis: Digging deeper than markets and hierarchies, in: Menard, Claude/Shirley, Mary M. (Hrsg.), Handbook of New Institutional Economics, Dordrecht, S. 819-848.

Perlmutter, Howard V./Heenan, David A. (1986), Cooperate to compete globally, in: Harvard Business Review, Vol. 64, S. 136-152.

Pfeffer, Jeffrey/Salancik, Gerald R. (1978), The External Control of Organizations: A Resource Dependence Perspective, New York.

Pfohl, Hans-Christian/Buse, Hans P. (1999), Organisationale Beziehungsfähigkeiten in komplexen kooperativen Beziehungen, in: Engelhard, Johann/Sinz, Elmar (Hrsg.), Kooperationen im Wettbewerb: Neue Formen und Gestaltungskonzepte im Zeichen von Globalisierung und Informationstechnologie, Wiesbaden, S. 269-300.

Phillips, Almarin (1960), A theory of interfirm organization, in: Quarterly Journal of Economics, Vol. 74, S. 602613.

Podolny, Joel M. (1993), A status-based model of market competition, in: American Journal of Sociology, Vol. 98, S. 829-872.

Podolny, Joel M. (1994), Market uncertainty and the social character of economic exchange, in: Administrative Science Quarterly, Vol. 39, S. 458-483.

Powell, Walter W. (1998), Learning from collaboration: Knowledge and networks in the biotechnology and pharmaceutical industries, in: California Management Review, Vol. 40, S. 228-240.

Powell, Walter W./Koput, Kenneth W./Smith-Doerr, Laurel (1996), Interorganizational collaboration and the locus of innovation: Networks of learning in biotechnology, in: Administrative Science Quarterly, Vol. 41, S. 116-145.

Rese, Mario (2006), Successful and sustainable business partnerships: How to select the right partners, in: Industrial Marketing Management, Vol. 35, S. 72-82.

Robinson, Patrick J./Faris, Charles W./Wind, Yoram (1967), Industrial Buying and Creative Marketing, Boston.

Santos, Filipe M./Eisenhardt, Kathleen M. (2009), Constructing markets and shaping boundaries: Entrepreneurial power in nascent fields, in: Academy of Management Journal, Vol. 52, S. 643-671.

Schauenberg, Bernd (2004), Marktversagen und Organisationsversagen, in: Schreyögg, Georg/von Werder, Axel (Hrsg.), Handwörterbuch Unternehmensführung und Organisation, 4. Aufl., Stuttgart, S. 820-828.

Schmid, Martin S. (2010), Der Wettbewerb zwischen Business Webs: Strategien konkurrierender Unternehmensnetzwerke im IPTV-Markt, Wiesbaden.

Schmidt, Axel/Kiefer, Clemens (2005), Kooperationen zwischen mittelständischen Unternehmen, in: Zentes, Joachim/ Swoboda, Bernhard/Morschett, Dirk (Hrsg.), Kooperationen, Allianzen und Netzwerke: Grundlagen - Ansätze Perspektiven, Wiesbaden, S. 1357-1381.

Schmidtchen, Dieter (2005), Wettbewerb und Kooperation (Co-opetition): Neues Paradigma für Wettbewerbstheorie und Wettbewerbspolitik, in: Zentes, Joachim/Swoboda, Bernhard/Morschett, Dirk (Hrsg.), Kooperationen, Allianzen und Netzwerke: Grundlagen - Ansätze - Perspektiven, Wiesbaden, S. 65-93.

Schubert, Werner/Küting, Karlheinz (1981), Unternehmungszusammenschlüsse, München.

Semlinger, Klaus (1993), Effizienz und Autonomie in Zulieferungsnetzwerken - Zum strategischen Gehalt von Kooperation, in: Staehle, Wolfgang H./Sydow, Jörg (Hrsg.), Managementforschung 3, Berlin, S. 309-354.

Sjurts, Insa (2000), Kollektive Unternehmensstrategie: Grundfragen einer Theorie kollektiven strategischen Handelns, Wiesbaden.

Smith, Adam (1976[1776]), An Inquiry into the Nature and Causes of the Wealth of Nations, Oxford.

Snow, Charles C./Miles, Raymond E./Coleman Jr., Henry J. (1992), Managing 21st century network organizations, in: Organizational Dynamics, Vol. 20, S. 5-20.

Stack, Martin/Gartland, Myles P. (2005), The repeal of prohibition and the resurgence of the national breweries: Productive efficiency or path creation?, in: Journal of Management History, Vol. 43, S. 420-432.

Steding, Rolf (2004), Das Konsortium - eine originelle Gestaltungsvariante für wirtschaftliche Vorhaben, in: Betrieb und Wirtschaft, 58. Jg., S. 108-109. 
Steiner, Florian (2004), Formation and Early Growth of Business Webs: Modular Product Systems in Network Markets, Heidelberg.

Sydow, Jörg (2001), Management von Unternehmungsnetzwerken - Auf dem Weg zu einer reflexiven Netzwerkentwicklung?, in: Howaldt, Jürgen/Kopp, RalflFlocken, Peter (Hrsg.), Kooperationsverbünde und regionale Modernisierung: Theorie und Praxis der Netzwerkarbeit, Wiesbaden, S. 79-101.

Sydow, Jörg/Duschek, Stephan/Möllering, Guido/Rometsch, Markus (2003), Kompetenzentwicklung in Netzwerken: Eine typologische Studie, Wiesbaden.

Sydow, Jörg/Möllering, Guido (2009), Produktion in Netzwerken: Make, Buy \& Cooperate, 2. Aufl., München.

Sydow, Jörg/Windeler, Arnold (1994), Über Netzwerke, virtuelle Integration und Interorganisationsbeziehungen, in: Sydow, Jörg/Windeler, Arnold (Hrsg.), Management interorganisationaler Beziehungen: Vertrauen, Kontrolle und Informationstechnik, Opladen, S. 1-21.

Sydow, Jörg/Windeler, Arnold (1999), Projektnetzwerke: Management von (mehr als nur) temporären Systemen, in: Engelhard, Johann/Sinz, Elmar (Hrsg.), Kooperation im Wettbewerb, Wiesbaden, S. 211-235.

Sydow, Jörg/Windeler, Arnold (Hrsg.) (2004), Organisation der Content-Produktion, Wiesbaden.

Sydow, Jörg/Windeler, Arnold/Wirth, Carsten (2002), Markteintritt als kollektiver Netzwerkeintritt - Internationalisierung der Fernsehproduktion in unreife Märkte, in: Die Betriebswirtschaft, 62. Jg., S. 459-473.

Teece, David J. (1992), Competition, cooperation, and innovation: Organizational arrangements for regimes of rapid technological progress, in: Journal of Economic Behavior \& Organization, Vol. 18, S. 1-25.

Uzzi, Brian (1997), Social structure and competition in interfirm networks: The paradox of embeddedness, in: Administrative Science Quarterly, Vol. 42, S. 35-67.

Vanhaverbeke, Wim/Norderhaven, Niels G. (2001), Competition between alliance blocks: The case of RISC microprocessor technology, in: Organization Studies, Vol. 22, S. 1-30.

von Weizsäcker, C. Christian (1999), Kooperation zwischen Konkurrenten aus Sicht der Wettbewerbspolitik, in: Engelhard, Johann/Sinz, Elmar (Hrsg.), Kooperation im Wettbewerb: Neue Formen und Gestaltungskonzepte im Zeichen von Globalisierung und Informationstechnologie, Wiesbaden, S. 479-493.

Wasserman, Stanley/Faust, Katherine (1994), Social Network Analysis: Methods and Applications, Cambridge.

Weber, Max (1980[1922]), Wirtschaft und Gesellschaft, 5. Aufl., Tübingen.

White, Harrison C. (1981), Where do markets come from?, in: American Journal of Sociology, Vol. 87, S. 517-547.

Wilhelm, Miriam (2009), Kooperation und Wettbewerb in Automobilzuliefernetzwerken: Erkenntnisse zum Management eines Spannungsverhältnisses aus Deutschland und Japan, Marburg.

Williamson, Oliver E. (1975), Markets and Hierarchies: Analysis and Antitrust Implications, New York.

Windeler, Arnold (2001), Unternehmungsnetzwerke: Konstitution und Strukturation, Wiesbaden.

Wirth, Carsten (2010), Reflexive Arbeitskräftewirtschaft: Strukturation, Projektnetzwerke und TV-Content-Produktion, München/Mering.

Wöhe, Günter/Döring, Ulrich (2008), Einführung in die Allgemeine Betriebswirtschaftslehre, 23. Aufl., München.

Wohlgemuth, Oliver (2002), Management netzwerkartiger Kooperationen: Instrumente für die unternehmensübergreifende Steuerung, Wiesbaden.

Wolters, Heiko (1995), Modul- und Systembeschaffung in der Automobilindustrie: Gestaltung der Kooperation zwischen europäischen Hersteller- und Zulieferunternehmen, Wiesbaden.

Zentes, Joachim/Swoboda, Bernhard (1999), Motive und Erfolgsgrößen internationaler Kooperation im Mittelstand, in: Die Betriebswirtschaft, 59. Jg., S. 44-60.

Zohlnhöfer, Werner (1991), Marktstruktur und funktionsfähiger Wettbewerb: Versuch der Erweiterung des Konzepts von Kantzenbach, in: Kantzenbach, Erhard/Molitor, Bruno/Mayer, Otto G. (Hrsg.), Hamburger Jahrbuch für Wirtschafts- und Gesellschaftspolitik 36, Tübingen, S. 71-86. 


\section{Summary}

This paper qualifies common conceptions of cooperation as a threat to competition or as a response to market failure. Instead, the paper argues that cartels, consortia and other forms of cooperation create the preconditions for market competition in a broader sense that is not restricted to competition between individual market actors for transactions but includes also competition between actors for partners and between networks of actors for transactions. It is especially in the phase of market emergence with a high level of uncertainty that strategic management can shape the horizons, intensities and dynamics of cooperative ties and thereby influence not only a firm's individual position in a new market but also the development of constitutive elements of the market as a whole. 